\title{
A variation norm Carleson theorem for vector-valued Walsh-Fourier series
}

\author{
Tuomas P. Hytönen, Michael T. Lacey and Ioannis Parissis
}

\begin{abstract}
We prove a variation norm Carleson theorem for WalshFourier series of functions with values in certain UMD Banach spaces, sharpening a recent result of Hytönen and Lacey. They proved the pointwise convergence of Walsh-Fourier series of $X$-valued functions under the qualitative hypothesis that $X$ has some finite tile type $q<\infty$, which holds in particular if $X$ is intermediate between another UMD space and a Hilbert space. Here we relate the precise value of the tile type index to the quantitative rate of convergence: tile type $q$ of $X$ is 'almost equivalent' to the $L^{p}$-boundedness of the $r$-variation of the Walsh-Fourier sums of any function $f \in L^{p}([0,1) ; X)$ for all $r>q$ and large enough $p$.
\end{abstract}

\section{Introduction}

The celebrated theorem of Carleson on the pointwise convergence of Fourier series [5] asserts that the partial sums of the Fourier series of an $L^{p}$-function converge almost everywhere to the function for $1<p<\infty$. The usual strategy to prove this result is to show that the Carleson operator, that is, the maximal partial sums of the Fourier series of a function, is bounded on the corresponding $L^{p}$-space. Subtle refinements of the Carleson theorem in [8] involve more refined notions than the $\ell^{\infty}$-norm of the partial sums. One of these notions is the variation norm Carleson theorem, proved in [20], which in particular shows the pointwise convergence of the Fourier series of $f$ without having to resort to a dense subset where this convergence can be easily exhibited. Weighted versions of the corresponding results for Fourier and Walsh-Fourier series have recently appeared in [11] and [10].

We point out that, in the scalar case, variational bounds for the Hilbert transform as well as for more general Calderón-Zygmund operators have been obtained for example in [3] and [4]. In [15] the authors study more singular operators such as averages along lower dimensional sets and truncations of singular integrals with

Mathematics Subject Classification (2010): Primary 42B20; Secondary 46E40.

Keywords: Pointwise convergence, variational norm, Walsh-Fourier series. 
rough kernels. However, there is no complete characterization of the CalderónZygmund kernels, say, that give rise to operators that obey variational bounds. The question is subtle since a variational bound immediately implies the pointwise converge of the truncations of singular integrals and it is known that this convergence fails for some operators. ${ }^{1}$ In the vector-valued setup information is even scarcer. For example it is natural to ask whether the Hilbert transform of a function with values in a UMD Banach space obeys variational bounds. This would be the vector-valued analog of the main result in [3] and, to the best of our knowledge, its validity is not currently known.

A necessary condition for the validity of Carleson's theorem for functions with values in a Banach space is known to be that the target Banach space $X$ has the UMD property: $X$-valued martingale differences converge unconditionally in the space. See for example [12], [25], and [24]. The first results on Carleson's theorem for vector-valued functions appear in [25] and [24] with the additional hypothesis that the target Banach space $X$ is a UMD space with an unconditional basis, or more generally, that it is a UMD lattice. The corresponding result for WalshFourier series was proved for UMD lattices in [29]. Recently in [21], Parcet, Soria, and $\mathrm{Xu}$ proved the weaker statement that the partial Fourier series of $f \in L^{p}(\mathbb{T} ; X)$, $p>1$, satisfy $S_{N} f(x)=o(\log \log N)$ for a.e. $x$, in general UMD spaces $X$, thus taking a step away from the lattice hypothesis. The first two authors of the current paper finally proved the vector valued Carleson theorem for all known examples of UMD spaces in [12]. Besides the UMD hypothesis, which is necessary, the proof in [12] is based on a certain key assumption on the UMD space, namely that it has finite tile type. The tile type assumption is a hypothesis of probabilistic flavor on the geometry of $X$, which is easily seen to be stronger than usual cotype, although we do not know at this moment if it is strictly stronger than the UMD property, which is known to imply finite cotype.

The tile type hypothesis was introduced in [13] where it is shown that finitetile type implies that the partial sums of Walsh-Fourier series converge to the function almost everywhere. Another hypothesis with a similar flavor was used in [12] to show the corresponding result in the trigonometric case. In these two papers, the prototypical examples of UMD Banach spaces with finite tile type are the intermediate spaces $X=[Y, H]_{\theta}$, that is, spaces $X$ which are complex interpolation spaces for some UMD Banach space $Y$ and a Hilbert space $H$. This class of Banach spaces includes for example all the UMD lattices but also the Schatten ideals $C_{p}, 1<p<\infty$, and in general all examples of UMD spaces currently known that are not necessarily function lattices. However the tile type turns out to be a qualitative hypothesis for the Carleson theorem: the exact value of the tile type is irrelevant, it is only needed that it is finite.

This changes dramatically when one looks at models for vector-valued bilinear Hilbert transforms, [14]. There the exact value of the tile types of the Banach spaces involved plays a crucial role in determining the range of inequalities one can prove, and in fact one needs to stay 'close enough' to the Hilbert space

\footnotetext{
${ }^{1}$ The study of variational bounds for quite general Calderón-Zygmund operators is addressed systematically in the paper [27] which appeared while the current paper was under review.
} 
tile type $q=2$ to get any bounds on the Walsh model of the bilinear Hilbert transform. ${ }^{2}$

In this paper we take up the investigation initiated in [13], and continued in [12] and [14], concerning vector-valued extensions of Carleson's theorem and related issues, where the lattice assumption is completely avoided. In particular, this paper is very much in the spirit of [13] since we study the variant of Carleson's theorem, due to Billard, [2], for Walsh-Fourier series. Building on the results from [13] and [12], our purpose is to investigate whether the finite tile type hypothesis is also necessary for the validity of a vector-valued Carleson theorem. We take a step in this direction by characterizing the UMD Banach spaces for which the finite tile type assumption is true in terms of the boundedness of a variational Carleson operator. In particular we show that a variation norm version of Carleson's theorem for vector-valued Walsh-Fourier series, in the spirit of [20] and [11], is valid if and only if the UMD Banach space satisfies the finite tile type hypothesis.

Furthermore, we manage to quantify the relation between the tile type of the Banach space and the variation index in the variational Carleson theorem. Although this is not possible in the usual formulation of the Carleson theorem, the variational variant gives the correct framework for such a quantification. Thus the variation index, which can be viewed as a quantification of the rate of convergence of the partial Walsh-Fourier series, is intimately related to the tile type of the UMD Banach space under consideration. We know from [12] that tile type implies Carleson's theorem. However we now see that the tile type condition is essentially characterized by the validity of a variation norm Carleson theorem on a UMD Banach space. The question whether Carleson's theorem is valid on an arbitrary UMD Banach space remains open however. Our method and general strategy of proof is along the lines of [13], [11] and [20], using the time-frequency analysis techniques and arguments introduced in [16].

The first thing we need to do in order to formulate our main results is to describe what is the variation norm of a sequence. This norm plays a central role throughout the paper.

Let $1 \leq r \leq \infty$ and $x=\left\{x_{n}\right\}_{n \in \mathbb{N}}$ be a sequence of elements of a Banach space $(X,|\cdot|)$. The $r$-variation of the sequence $x$ is defined as

$$
\|x\|_{\mathcal{V}^{r}(X)}:=\sup _{K} \sup _{N_{0}<\cdots<N_{K}}\left(\sum_{j=1}^{K}\left|x_{N_{j}}-x_{N_{j-1}}\right|^{r}\right)^{1 / r},
$$

with the usual modification when $r=\infty$. Observe that the $\mathcal{V}^{\infty}$-norm is essentially the $\ell^{\infty}$-norm. If $\left\{f_{n}\right\}_{n \in \mathbb{N}}$ is a sequence of $X$-valued functions, $f_{n}: \mathbb{R}_{+} \rightarrow X$, we will also write

$$
\left\|f_{n}\right\|_{L^{p}\left(\mathbb{R}_{+} ; \mathcal{V}^{r}(X)\right)}:=\left(\int_{\mathbb{R}_{+}}\left\|f_{n}(x)\right\|_{\mathcal{V}^{r}(X)}^{p} d x\right)^{1 / p} .
$$

\footnotetext{
${ }^{2}$ There is another striking detail, that one can formulate vector-valued results that include non-UMD Banach spaces; see Silva [26].
} 
Now, for $f \in L^{p}([0,1) ; X)$ for some $1<p<\infty$, consider the partial Walsh sums

$$
S_{N} f(x):=\sum_{n=0}^{N-1}\left\langle f, w_{n}\right\rangle w_{n}(x),
$$

where $w_{n}$ is the $n$th Walsh function on $[0,1)$. Precise definitions will be given in Section 3. The variational Carleson operator is the nonlinear operator on the left below. As we shall see in detail in $\S 5$ it can be controlled in the form

$$
\left\|S_{N} f(x)\right\|_{\mathcal{V}^{r}} \lesssim C_{r, \mathbf{P}_{1}} f(x)+\tilde{C}_{r, \mathbf{P}_{2}} f(x)
$$

where $C_{r, \mathbf{P}_{1}}$ and $\tilde{C}_{r, \mathbf{P}_{2}}$ are linear operators defined in (5.1) in terms of notions from time-frequency analysis which will be introduced in $\S 3$. The point of introducing these operators is that they linearize and control the variational Carleson operator, and are more amenable to time-frequency analysis. Our main theorem characterizes the tile type of a Banach space in terms of the boundedness of the operator $C_{r, \mathbf{P}_{1}}$ or the operator $\tilde{C}_{r, \mathbf{P}_{2}}$.

Theorem 1.1. Let $q \in[2, \infty)$ and let $X$ be a Banach space. Suppose that the operator $C_{r, \mathbf{P}}$, or the operator $\tilde{C}_{r, \mathbf{P}}$, satisfies

$$
\left\|C_{r, \mathbf{P}} f\right\|_{L^{r}\left(\mathbb{R}_{+} ; X\right)} \lesssim\|f\|_{L^{r}\left(\mathbb{R}_{+} ; X\right)},
$$

whenever $q<r<\infty$. Then $X$ has tile type $\tau$ for all $\tau>q$ and, a fortiori, $X$ has cotype $\tau$ for all $\tau>q$.

Conversely, suppose that the Banach space $X$ has tile type $\tau$ for all $\tau>q$. Then for every $f \in L^{p}\left(\mathbb{R}_{+} ; X\right)$,

$$
\left\|C_{r, \mathbf{P}_{1}} f\right\|_{L^{p}\left(\mathbb{R}_{+} ; X\right)}+\left\|\tilde{C}_{r, \mathbf{P}_{2}} f\right\|_{L^{p}\left(\mathbb{R}_{+} ; X\right)} \lesssim\|f\|_{L^{p}\left(\mathbb{R}_{+} ; X\right)},
$$

for $\max \left(q, p^{\prime}(q-1)\right)<r<\infty$, with the implicit constant depending only upon $p, r, q$, and the space $X$.

As a corollary we conclude a variational norm version of Carleson's theorem for vector-valued Walsh-Fourier series:

Theorem 1.3. Given $q \in[2, \infty)$, let $X$ be a Banach space which has tile type $\tau$ for all $\tau>q$. Suppose that

$$
\max \left(q, p^{\prime}(q-1)\right)<r<\infty .
$$

Then for every $f \in L^{p}([0,1) ; X)$,

$$
\left\|S_{N} f\right\|_{L^{p}\left([0,1) ; \mathcal{V}^{r}(X)\right)} \lesssim\|f\|_{L^{p}([0,1) ; X)},
$$

with the implicit constant depending only upon $p, r, q$, and the space $X$.

Observe that for $q=2$ the restriction on the integrability exponent becomes $p>r^{\prime}$ which is necessary in the scalar case of the Fourier analog of Theorem 1.3, [20]. Here we get a condition that becomes more stringent as $X$ 'moves away' from the Hilbert space case, quantified by the tile type. 
In the special case that $X$ is an intermediate space, that is $X=[Y, H]_{\theta}$ is a complex interpolation space for some UMD Banach space $Y$ and a Hilbert space $H$, and $0<\theta<1$, it was shown in [13] that $X$ has tile type $q=2 / \theta$. Thus Theorem 1.3 immediately applies to all intermediate spaces of this type. However, arguing directly by interpolation we get a slightly stronger theorem:

Theorem 1.4. Suppose that $X:=[Y, H]_{\theta}, 0<\theta<1$, is a complex interpolation space between a UMD Banach space $Y$ and a Hilbert space $X$. Set $q:=2 / \theta$. Suppose that

$$
\max \left(q, p^{\prime} q / 2\right)<r<\infty .
$$

Then for every $f \in L^{p}([0,1) ; X)$ we have that

$$
\left\|S_{N} f\right\|_{L^{p}\left([0,1) ; \mathcal{V}^{r}(X)\right)} \lesssim\|f\|_{L^{p}([0,1) ; X)} .
$$

where the implicit constant depends only on $p, r, q$, and the space $X$.

So far the only Banach spaces which are known to have finite tile type are exactly the complex interpolation spaces of Theorem 1.4. From this point of view, the weaker Theorem 1.3 seems uninteresting in comparison to Theorem 1.4. However, the formulation of Theorem 1.3 only assumes finite tile type. This provides an indication that the finite tile type of a Banach space $X$ could be a strictly weaker hypothesis than that of $X$ being a complex interpolation space.

The Fourier scalar variational Carleson theorem is related to a number of topics, including variational estimates for singular integrals, [15]; refined estimates in ergodic theory, [8], [7]; maximal inequalities, [19], and approaches to extensions of results of Christ-Kiselev, [6], in spectral theory; see [20], Appendix C. Some of these continue to be under active development, [18], [9]. At some point, these topics might be ripe for investigation in the vector-valued case.

The rest of the paper is organized as follows. In section 3 we introduce tiles and trees in the time-frequency plane and define the corresponding wave packets in terms of appropriate Walsh functions. In section 4 we review the definition of the tile type of a Banach space, adjusted to the needs of the present paper. This definition is slightly weaker than the one in [13] but philosophically it is the same. We also discuss several structural properties of the trees that one needs to consider in the definition of the tile type and show that tile type $q$ implies martingale cotype $q$ for any Banach space $X$. We also recall the vector-valued version of Lépingle's inequality which will play an important role later in the proof. In section 5 we linearize the variational Carleson operator and introduce its variants $C_{r, \mathbf{P}}$ which characterize the tile type of the Banach space $X$. We eventually linearize all our operators and reduce the main theorem to the statements of Propositions 5.4 and 5.6. In Sections 6 to 8 we introduce all the necessary machinery from timefrequency analysis and prove Proposition 5.6. The last section 9 is devoted to the proof of Theorem 1.4. The proof uses complex interpolation between the full range of $r$-variation inequalities, valid in any Hilbert space, and the $\infty$-variation bounds for intermediate spaces from [13]. 


\section{Notation}

Throughout the text $c$ and $C$ will denote generic positive constants that might change even in the same line of text. We write $A \lesssim B$ if $A \leq c B$ for some numerical constant $c>0$ and $A \approx B$ if $A \lesssim B$ and $B \lesssim A$. In this paper the implicit constants that appear in various estimates may depend on the variation index $r$, the integrability index $p$, the tile type index $q$ and the space $X$ itself, but we typically suppress this dependence since it is of no importance. We denote by $\mathbb{N}$ the set of nonnegative integers and by $\mathbb{R}_{+}$the set of nonnegative real numbers. Finally the dyadic subintervals of the positive real line are denoted by $\mathcal{D}$. These are the intervals of the form $\left[n 2^{k},(n+1) 2^{k}\right)$ where $n \in \mathbb{N}$ and $k \in \mathbb{Z}$. For any interval $\omega$ with endpoints $a<b$ we use the standard notation $\stackrel{\circ}{\omega}:=(a, b)$ for its interior as well as the notations $[\omega):=[a, b)$ and $(\omega]:=(a, b]$. If no special notation is used by convention we use $\omega=[\omega)=[a, b)$.

For $k \in \mathbb{Z}$ we denote by $\mathbb{E}_{k}$ the conditional expectation with respect to dyadic intervals of length $2^{k}$ :

$$
\mathbb{E}_{k} f(x):=\sum_{I \in \mathcal{D},|I|=2^{k}} \frac{\mathbf{1}_{I}(x)}{|I|} \int_{I} f(y) d y=: \sum_{I \in \mathcal{D},|I|=2^{k}} \mathbb{E}_{I} f(x) ;
$$

the dyadic maximal function is then $M f(x):=\sup _{k}\left|\mathbb{E}_{k} f(x)\right|$. Finally, we denote by BMO the dyadic BMO space on the positive real line, equipped with the norm

$$
\|f\|_{\mathrm{BMO}\left(\mathbb{R}_{+} ; X\right)}=\|f\|_{\mathrm{BMO}}:=\sup _{I \in \mathcal{D}} \frac{1}{|I|} \int_{I}\left|f(x)-\langle f\rangle_{I}\right| d x,
$$

where $\langle f\rangle_{I}:=\frac{1}{|I|} \int_{I} f$. Note that, throughout the text, the notation $|\cdot|$ is used both for the absolute value as well as for the norm of the Banach space $X$, depending on context.

\section{Walsh wave packets, tiles, and trees}

A tile $P$ is a dyadic rectangle of area 1 in the time-frequency plane $\mathbb{R}_{+} \times \mathbb{R}_{+}$, namely

$$
P=I_{P} \times \omega_{P}=I_{P} \times \frac{1}{\left|I_{P}\right|}[n, n+1), \quad n \in \mathbb{N}, \quad I_{P} \in \mathcal{D},
$$

If $P$ and $P^{\prime}$ are tiles we write $P \leq P^{\prime}$ if $I_{P} \subset I_{P^{\prime}}$ and $\omega_{P^{\prime}} \subset \omega_{P}$. Likewise, a bitile $P$ is a dyadic rectangle of area 2 :

$$
P=I_{P} \times \omega_{P}=I_{P} \times \frac{2}{\left|I_{P}\right|}[n, n+1)=\bigcup_{v=0}^{1} I_{P} \times \frac{1}{\left|I_{P}\right|}[2 n+v, 2 n+v+1) .
$$

Thus each bitile $P=P_{d} \cup P_{u}$ has a 'down-part' and an 'up-part' which are also dyadic. Furthermore we write

$$
P \leq_{u} P^{\prime} \stackrel{\text { def }}{\Longleftrightarrow} P_{u} \leq P_{u}^{\prime} \text { and } P \leq_{d} P^{\prime} \stackrel{\text { def }}{\Longleftrightarrow} P_{d} \leq P_{d}^{\prime} .
$$


The partial order for bitiles $P$ and $P^{\prime}$ is then defined as $P \leq P^{\prime} \stackrel{\text { def }}{\Longleftrightarrow} P \leq_{u} P^{\prime}$ or $P \leq_{d} P^{\prime}$. A tree $\mathbf{T}$ is a collection of bitiles $P$ for which there exists a top bitile $T$, which is not necessarily part of the collection, such that $P \leq T$ for all $P \in \mathbf{T}$. Note that in general a top of a tree is not uniquely defined. Similarly we say that $\mathbf{T}$ is an up-tree if $P \leq_{u} T$ for all $P \in \mathbf{T}$ and some top $T$ and a down-tree if $P \leq_{d} T$ for all $P \in \mathbf{T}$. Trivially any tree can be decomposed into an up-tree and a down-tree:

$$
\mathbf{T}=\mathbf{T}_{u} \cup \mathbf{T}_{d}, \quad \mathbf{T}_{u} \stackrel{\text { def }}{=}\left\{P \in \mathbf{T}: P \leq_{u} T\right\}, \quad \mathbf{T}_{d} \stackrel{\text { def }}{=}\left\{P \in \mathbf{T}: P \leq_{d} \mathbf{T}\right\} .
$$

The Rademacher functions are defined as

$$
r_{i}(x):=\operatorname{sgn} \sin \left(2 \pi \cdot 2^{i} x\right)=\sum_{k \in \mathbb{N}}\left(\mathbf{1}_{2^{-i}[k, k+1 / 2)}(x)-\mathbf{1}_{2^{-i}[k+1 / 2, k+1)}(x)\right) .
$$

If $n \in \mathbb{N}$ has the binary expansion

$$
n=\sum_{i=0}^{\infty} n_{i} 2^{i}, \quad n_{i} \in\{0,1\},
$$

we define the $n$th Walsh function to be

$$
w_{n}(x):=\prod_{i=0}^{\infty} r_{i}(x)^{n_{i}} .
$$

Observe that we recover the Rademacher functions from the Walsh functions by $w_{2^{i}}(x)=r_{i}(x)$. It is well known and easy to see that the restrictions $\left\{w_{n} \mathbf{1}_{[0,1)}\right\}_{n \in \mathbb{N}}$ constitute an orthonormal basis of $L^{2}(0,1)$.

With these definitions at hand we now associate to each tile $P \subset \mathbb{R}_{+} \times \mathbb{R}_{+}$a wave packet $w_{P}$ as follows. First we write the tile $P$ with respect to its time and frequency components:

$$
P=I_{P} \times \omega_{P}=I_{P} \times \frac{1}{\left|I_{P}\right|}[n, n+1), \quad I_{P} \in \mathcal{D}, \quad n \in \mathbb{N} .
$$

The wave packet $w_{P}$ is now defined as

$$
w_{P}(x):=\frac{1}{\left|I_{P}\right|^{1 / 2}} \mathbf{1}_{I_{P}}(x) w_{n}\left(\frac{x}{\left|I_{P}\right|}\right)=: \frac{1}{\left|I_{P}\right|^{1 / 2}} w_{P}^{\infty}(x) .
$$

Observe that $w_{P}$ is $L^{2}$-normalized while $w_{P}^{\infty}$ is $L^{\infty}$-normalized, hence the superscript $\infty$. The Haar functions arise in the special case of wave packets corresponding to tiles of the form $P=I \times|I|^{-1}[1,2)$ :

$$
h_{I}(x):=\frac{1}{|I|^{1 / 2}} \mathbf{1}_{I}(x) r_{0}\left(\frac{x}{|I|}\right)=w_{I \times|I|^{-1}[1,2)}(x) .
$$

Given a bitile $P=P_{d} \cup P_{u}$ we use the notations $w_{P_{u}}$ and $w_{P_{d}}$ for the wave packets associated with the up-part and the down-part of the bitile $P$, respectively.

If a collection of bitiles arises from a single up-tree then the following lemma gives a very useful description of the wave packets in terms of the simpler Haar functions. This lemma is taken from Lemma 2.2 in [13], where we also refer the reader for the proof. 
Lemma 3.2. Let $\mathbf{T}$ be an up-tree with top $T$. For all $P \in \mathbf{T}$ we have

$$
w_{P_{d}}(x)=\epsilon_{P T} \cdot w_{T_{u}}^{\infty}(x) \cdot h_{I_{P}}(x),
$$

where $w_{T_{u}}^{\infty}$ is the $L^{\infty}$-normalized wave packet associated to the up-part of the top $T$ and $\epsilon_{P T} \in\{-1,+1\}$ is a constant factor that depends only on $P$ and $T$. In particular we have that

$$
\left\langle f, w_{P_{d}}\right\rangle w_{P_{d}}=\left\langle f \cdot w_{T_{u}}^{\infty}, h_{I_{P}}\right\rangle h_{I_{P}} \cdot w_{T_{u}}^{\infty} .
$$

If $\mathbf{T}$ is a down-tree with top $T$ we have a symmetric statement:

$$
w_{P_{u}}(x)=\tilde{\epsilon}_{P T} \cdot w_{T_{d}}^{\infty}(x) \cdot h_{I_{P}}(x) .
$$

\section{Tile type and cotype of a Banach space}

The notion of the tile type of a Banach space $X$ was introduced in [13] to show that if a Banach space $X$ has finite tile type then Carleson's theorem for Walsh-Fourier series is true for $X$-valued functions. More recently it was shown in [12] that a variant of the tile type, adapted to Fourier wave packets, also implies Carleson's theorem for the trigonometric system. Here we give a definition which is similar in spirit to that in $\S 3$ of [13]. The main difference is that we only consider very special collections of trees in the definition of tile type. These are essentially the trees generated by the selection algorithm in the size lemma, Lemma 7.5, and that lemma is the only place in the proof where the tile type hypothesis is needed. In fact the reader is encouraged to briefly go through the statement and proof of the size lemma in order to gain some intuition on the definition that follows. The reason for giving this weaker but more complicated definition of tile type is that it allows us to prove a partial converse of the variational Carleson theorem in Proposition 5.4, namely that the $r$-variational boundedness of a Carleson-type operator implies that the space $X$ necessarily has tile type $\tau$ for all $\tau>r$.

\subsection{Good collections of trees and tile type of a Banach space}

We now describe the collections of trees that we want to consider in the definition of tile type. Let $\mathcal{T}=\left\{\mathbf{T}_{j}\right\}_{j}$ be a finite collection of up-trees, each consisting of finitely many bitiles, and set

$$
\mathbf{P}:=\left\{P: P \in \mathbf{T}_{j} \text { for some } j\right\}=\cup_{j} \mathbf{T}_{j} .
$$

Denote by $c(I)$ the center of some dyadic interval $I \in \mathcal{D}$. We will call the collection $\mathcal{T} u(p)$-good if it has the following property:

There is a reordering of the trees $\left\{\mathbf{T}_{j}\right\}_{j}$ and a choice of corresponding tops $\left\{T_{j}\right\}_{j}$ such that $\left\{c\left(\omega_{T_{j}}\right)\right\}_{j}$ is an increasing sequence and

$$
\mathbf{T}_{j}=\left\{P \in \mathbf{P}: P \leq{ }_{u} T_{j} \text { and } P \not \leq T_{k} \text { for all } k<j\right\} .
$$


There is a symmetric definition of a good collection of down-trees, namely a collection $\mathcal{T}$ of down-trees will be called $d($ own $)$-good if there is a reordering of the trees $\left\{\mathbf{T}_{j}\right\}_{j}$ and a choice of corresponding tops $\left\{T_{j}\right\}_{j}$ such that $\left\{c\left(\omega_{T_{j}}\right)\right\}_{j}$ is a decreasing sequence and

$$
\mathbf{T}_{j}=\left\{P \in \mathbf{P}: P \leq_{d} T_{j} \text { and } P \not \leq T_{k} \text { for all } k<j\right\} .
$$

We say that a Banach space $X$ has $u$-tile type $q$ if the estimate

$$
\left(\sum_{\mathbf{T} \in \mathcal{T}}\left\|\sum_{P \in \mathbf{T}}\left\langle f, w_{P_{d}}\right\rangle w_{P_{d}}\right\|_{L^{q}\left(\mathbb{R}_{+} ; X\right)}^{q}\right)^{1 / q} \lesssim\|f\|_{L^{q}\left(\mathbb{R}_{+} ; X\right)},
$$

holds uniformly for all $u$-good collections $\mathcal{T}$. Similarly, we say that a Banach space $X$ has $d$-tile type $q$ if

$$
\left(\sum_{\mathbf{T} \in \mathcal{T}}\left\|\sum_{P \in \mathbf{T}}\left\langle f, w_{P_{u}}\right\rangle w_{P_{u}}\right\|_{L^{q}\left(\mathbb{R}_{+} ; X\right)}^{q}\right)^{1 / q} \lesssim\|f\|_{L^{q}\left(\mathbb{R}_{+} ; X\right)},
$$

uniformly, for all $d$-good collections $\mathcal{T}$. It is actually not hard to see that the two definitions of tile type, namely the one given with respect to $u$-good collections and the one given with respect to $d$-good collections, are equivalent. This is the content of the following lemma.

Lemma 4.1. A Banach space $X$ has u-tile type $q$ if and only if it has d-tile type $q$.

Proof. Assume that $X$ has $u$-tile type $q$ and let $\mathcal{T}$ be a $d$-good collection of downtrees. Fix a choice of tops $\left\{T_{j}\right\}_{j}$ ordered so that the sequence of centers $\left\{c\left(\omega_{T_{j}}\right)\right\}_{j}$ is decreasing and

$$
\mathbf{T}_{j}=\left\{P \in \mathbf{P}: P \leq_{d} T_{j} \text { and } P \not \leq T_{k} \text { for all } k<j\right\} .
$$

Let $\mathbf{P}$ be the collection of all tiles in $\mathcal{T}$ and define $2^{N}:=\sup _{P \in \mathbf{P}} \sup \omega_{P}$. For any bitile $P \in \mathbf{P}, P=I_{P} \times\left|I_{P}\right|^{-1}[n, n+2)$, we define the transformation:

$$
P \mapsto \tilde{P}:=I_{P} \times\left[2^{N}-(n+2)\left|I_{P}\right|^{-1}, 2^{N}-n\left|I_{P}\right|^{-1}\right) .
$$

By the choice of $N$ the transformation above maps bitiles $P \subset \mathbb{R}_{+} \times \mathbb{R}_{+}$into bitiles $\tilde{P} \subset \mathbb{R}_{+} \times \mathbb{R}_{+}$. Observe also that the down-part of a bitile $P$ is mapped to the up-part of the bitile $\tilde{P}$ and vice versa. Transforming the tops $\left\{T_{j}\right\}_{j}$ accordingly we obtain a collection $\tilde{\mathcal{T}}$, consisting of up-trees, and a sequence of tops $\left\{\tilde{T}_{j}\right\}_{j}$ which together form a $u$-good collection. By the assumption that $X$ has $u$-tile type $q$ we thus get

$$
\left(\sum_{\tilde{\mathbf{T}} \in \tilde{\mathcal{T}}}\left\|\sum_{\tilde{P} \in \tilde{\mathbf{T}}}\left\langle f, w_{\tilde{P}_{d}}\right\rangle w_{\tilde{P}_{d}}\right\|_{L^{q}\left(\mathbb{R}_{+} ; X\right)}^{q}\right)^{1 / q} \lesssim\|f\|_{L^{q}\left(\mathbb{R}_{+} ; X\right)} .
$$

Once this estimate is established, the specific choice of tops $\left\{T_{j}\right\}_{j}$ is no longer relevant. For each tree $\mathbf{T}_{j}$ it is clear that we can choose a top $S_{j}$ with $\left|I_{S_{j}}\right|=$ 
$\max _{\ell}\left|I_{T_{\ell}}\right|$. Thus the number $\left|I_{S_{j}}\right|$ does not depend on $j$. Applying Lemma 3.2 to the tiles $\tilde{P}$ belonging to the up-tree $\tilde{\mathbf{T}}$ with top $\tilde{S}$, we have

$$
w_{\tilde{P}_{d}}(x)=\tilde{\epsilon}_{P S} \cdot h_{I_{S}}(x) \cdot w_{\tilde{S}_{u}}^{\infty}(x) \quad \text { and } \quad w_{P_{u}}(x)=\epsilon_{P S} \cdot h_{I_{S}}(x) \cdot w_{S_{d}}^{\infty}(x) .
$$

Define $2^{m}:=2^{N}\left|I_{S}\right|$ and note that this number does not depend on the specific choice of tree $\mathbf{T}$. In order to derive a relation between $w_{\tilde{S}_{u}}^{\infty}$ and $w_{S_{d}}^{\infty}$ we write $S=I_{S} \times\left|I_{S}\right|^{-1}[n, n+2)$ so that $S_{d}=I_{S} \times\left|I_{S}\right|^{-1}[n, n+1)$ and $\tilde{S}_{u}=I_{S} \times$ $\left|I_{S}\right|^{-1}\left[2^{m}-n-1,2^{m}-n\right)$. We have

$$
\begin{aligned}
w_{\tilde{S}_{u}}^{\infty}(x) & =\mathbf{1}_{I_{S}}(x) w_{2^{m}-n-1}\left(x /\left|I_{S}\right|\right)=\mathbf{1}_{I_{S}}(x) w_{2^{m}-1}\left(x /\left|I_{S}\right|\right) w_{n}\left(x /\left|I_{S}\right|\right) \\
& =w_{2^{m}-1}\left(x /\left|I_{S}\right|\right) \cdot w_{S_{d}}^{\infty}(x)=: \phi_{\mathbf{P}}(x) \cdot w_{S_{d}}^{\infty}(x),
\end{aligned}
$$

where $\phi_{\mathbf{P}}$ is a unimodular function that depends only on the collection $\mathbf{P}$. Thus

$$
\begin{aligned}
\|f\|_{L^{q}\left(\mathbb{R}_{+} ; X\right)} & \gtrsim\left(\sum_{\tilde{\mathbf{T}} \in \tilde{\mathcal{T}}}\left\|\sum_{\tilde{P} \in \tilde{\mathbf{T}}}\left\langle f, w_{\tilde{P}_{d}}\right\rangle w_{\tilde{P}_{d}}\right\|_{L^{q}\left(\mathbb{R}_{+} ; X\right)}^{q}\right)^{1 / q} \\
& =\left(\sum_{\mathbf{T} \in \mathcal{T}}\left\|\sum_{P \in \mathbf{T}}\left\langle f \phi_{\mathbf{P}}, w_{P_{u}}\right\rangle w_{P_{u}} \phi_{\mathbf{P}}\right\|_{L^{q}\left(\mathbb{R}_{+} ; X\right)}^{q}\right)^{1 / q} .
\end{aligned}
$$

Replacing $f$ by $f \phi_{\mathbf{P}}^{-1}$ in the previous estimate we conclude that $X$ has $d$-tile type $q$. The proof of the reverse implication is completely symmetric.

Remark 4.2. In view of Lemma 4.1 we will henceforth say that a Banach space $X$ has tile type $q$ whenever it has $u$-tile type or $d$-tile type $q$. We will also talk about good collections $\mathcal{T}$ without specifying whether we are talking about $u$-good or $d$ good collections. Furthermore, it is obvious that if a collection of trees can be split into a finite number $k$ of good collections then the tile type inequality still holds for the original collection with some different constant depending on $k$. We will then say that $\mathcal{T}$ is a $k$-good collection, or just a good collection if it is clear that the number $k$ does not depend on anything interesting.

The following lemma gathers some useful properties of good collections $\mathcal{T}$ and is the main ingredient in the proof of the partial converse in Proposition 5.4.

Lemma 4.3. Let $\mathcal{T}=\left\{\mathbf{T}_{j}\right\}_{j=1}^{M}$ be a good collection of up-trees and denote by $\mathbf{P}$ the set of all bitiles in $\mathcal{T}$. Let $\left\{T_{j}\right\}_{j=1}^{M}$ be the collection of the corresponding tops from the definition a good collection, ordered so that $\left\{c\left(\omega_{T_{j}}\right)\right\}_{j}$ is increasing. We have the following properties:

(i) The down-parts of the bitiles in $\mathcal{T}$ are disjoint:

$$
\text { if } P, P^{\prime} \in \mathbf{P} \text { and } P \neq P^{\prime} \text { then } P_{d}^{\prime} \cap P_{d}=\emptyset \text {. }
$$

(ii) Let $k(j, x):=\max _{k}\left\{1 \leq k \leq j: I_{T_{k}} \ni x\right\}$ with the understanding that $\max \emptyset$ $:=0$. For $j \in\{1,2, \ldots, M\}$ define the measurable functions $N_{j}: \mathbb{R}_{+} \rightarrow \mathbb{R}_{+}$ 
as $N_{j}(x):=c\left(\omega_{T_{k(j, x)}}\right)$. Let us also set $N_{0}(x) \equiv 0$ for convenience. For any fixed $x \in \mathbb{R}_{+}$the sequence $\left\{N_{j}(x)\right\}_{j}$ is increasing. Furthermore, for each $j$ and $x \in \mathbb{R}_{+}$we have

$$
\left\{P \in \mathbf{T}_{j}: I_{P} \ni x\right\}=\left\{P \in \mathbf{P}: I_{P} \ni x, N_{j}(x) \in\left[\omega_{P_{u}}\right) \text { and } N_{j-1}(x) \notin \stackrel{\circ}{\omega}_{P}\right\} .
$$

(iii) For $1 \leq r<\infty$ we have

$$
\begin{aligned}
\sum_{j}\left\|\sum_{P \in \mathbf{T}_{j}}\left\langle f, w_{P_{d}}\right\rangle w_{P_{d}}\right\|_{L^{r}\left(\mathbb{R}_{+} ; X\right)}^{r} & \\
& =\int \sum_{j}\left|\sum_{P \in \mathbf{P}}\left\langle f, w_{P_{d}}\right\rangle w_{P_{d}}(x) \mathbf{1}_{\left\{N_{j}(x) \in\left[\omega_{P_{u}}\right), N_{j-1}(x) \notin \dot{\omega}_{P}\right\}}\right|^{r} d x .
\end{aligned}
$$

Proof. For (i) observe that if two bitiles $P, P^{\prime} \in \mathbf{P}$ belong to the same tree $\mathbf{T} \in \mathcal{T}$ then we always have $P_{d} \cap P_{d}^{\prime}=\emptyset$ since $\mathbf{T}$ is an up-tree. Suppose now that $P \in \mathbf{T}_{j}$ and $P^{\prime} \in \mathbf{T}_{k}$ where $\mathbf{T}_{j}$ and $\mathbf{T}_{k}$ are two different trees in $\mathcal{T}$. Assume, with the aim of obtaining a contradiction, that $P_{d} \cap P_{d}^{\prime} \neq \emptyset$ so that $I_{P} \cap I_{P^{\prime}} \neq \emptyset$ and $\omega_{P_{d}} \cap \omega_{P^{\prime} d} \neq \emptyset$. Then we have for example that $\omega_{P_{d}} \subseteq \omega_{P^{\prime}{ }_{d}}$. However, since $P$ and $P^{\prime}$ are different tiles we must actually have that $\omega_{P} \subseteq \omega_{P_{d}^{\prime}}$. Thus

$$
\omega_{T_{j}} \subseteq \omega_{P} \subseteq \omega_{P_{d}^{\prime}} \subset \omega_{P^{\prime}} \text { and } \emptyset \neq I_{P} \cap I_{P^{\prime}} \subseteq I_{T_{j}} \cap I_{P^{\prime}}
$$

which implies that $P^{\prime} \leq T_{j}$. We then get the following inequality for the centers of $\omega_{T_{j}}$ and $\omega_{T_{k}}$ :

$$
c\left(\omega_{T_{j}}\right)<\sup \omega_{T_{j}} \leq \sup \omega_{P} \leq \sup \omega_{P_{d}^{\prime}}=\inf \omega_{P_{u}^{\prime}} \leq \inf \omega_{T_{k, u}}=c\left(\omega_{T_{k}}\right) .
$$

By the definition of a good collection we thus have that $j<k$ so that $P^{\prime} \not \leq T_{j}$, a contradiction.

We now prove (ii). First note that for every fixed $x \in \mathbb{R}_{+}$the sequence $\left\{N_{j}(x)\right\}_{j}$ is increasing as a composition of increasing functions of $j$. In order to prove the main claim in (ii) we fix $x \in \mathbb{R}_{+}$and $j \in\{1 \ldots, M\}$ and define the collections of bitiles

$$
\begin{aligned}
& S(j, x):=\left\{P \in \mathbf{T}_{j}: I_{P} \ni x\right\}, \\
& B(j, x):=\left\{P \in \mathbf{P}: I_{P} \ni x, N_{j}(x) \in \omega_{P_{u}}, \quad N_{j-1}(x) \notin \stackrel{\circ}{\omega}_{P}\right\},
\end{aligned}
$$

where $N_{j}(x)$ is as in the statement of the lemma. We claim that $S(j, x)=B(j, x)$. If $x \notin I_{T_{j}}$ then both collections are empty; for $S(j, x)$ this is because $x \notin I_{T_{j}} \supseteq I_{P}$ while for $B(j, x)$ because $N_{j-1}(x)=N_{j}(x)$ in this case.

It remains to verify the claim when $x \in I_{T_{j}}$ in which case $N_{j}(x)=c\left(\omega_{T_{j}}\right)$ and $N_{j-1}(x) \in\left\{0, c\left(\omega_{T_{k(j-1, x)}}\right)\right\}$.

Let $P \in S(j, x)$. Then $x \in I_{P} \subseteq I_{T_{j}}$ and by the definition of a good collection we have that $P \leq_{u} T_{j}$ and $P \not \leq T_{k}$ for any $k \leq j-1$. The condition $P \leq_{u} T_{j}$ implies that $N_{j}(x)=c\left(\omega_{T_{j}}\right) \in \omega_{P_{u}}$. If $x \notin \cup_{\ell \leq j-1} I_{T_{\ell}}$ then $N_{j-1}(x)=0$ which is never in the interior of any frequency interval thus $N_{j-1}(x) \notin \dot{\circ}_{P}$ in this case. On 
the other hand if $x \in \cup_{\ell \leq j-1} I_{T_{\ell}}$ we have that $x \in I_{T_{k(j-1, x)}}$ and $P \not \leq T_{k(j-1, x)}$ since $k(j-1, x)<j$. Since $x \in I_{P} \cap I_{T_{k(j-1, x)}} \neq \emptyset$ we must have $\omega_{T_{k(j-1, x)}} \nsubseteq \omega_{P}$ and thus $N_{j-1}(x)=c\left(\omega_{T_{k(j-1, x)}}\right) \notin \dot{\circ}_{P}$. This proves the inclusion $S(j, x) \subseteq B(j, x)$.

For the opposite inclusion assume that $P \in B(j, x)$. Since $x \in I_{T_{j}}$ we have that $N_{j}(x)=c\left(\omega_{T_{j}}\right) \in \omega_{P_{u}}$ which is equivalent to $\omega_{T_{j, u}} \subseteq \omega_{P_{u}}$. Since $x \in I_{P} \cap I_{T_{j}}$ this shows that $P \leq_{u} T_{j}$. Now it is not hard to see that $P \not \leq T_{\ell}$ whenever $\ell<j$. Indeed suppose that we had $P \leq T_{\ell}$ for some $\ell<j$. This would imply that $x \in I_{P} \subseteq I_{T_{\ell}}$ and thus $\ell \leq k(j-1, x)<j$. Furthermore we would have $\omega_{T_{\ell}}, \omega_{T_{j}} \subseteq \omega_{P}$ so by the convexity of the interval $\omega_{P}$ and the fact that the sequence $\left\{c\left(\omega_{T_{j}}\right)\right\}_{j}$ is increasing we would conclude that $N_{j-1}(x)=c\left(\omega_{T_{k(j-1, x)}}\right) \in \stackrel{\circ}{\omega}_{P}$, contradicting the second condition in the definition of $B(j, x)$. This proves the inclusion $B(j, x) \subseteq S(j, x)$ and thus concludes the proof of (ii).

Finally part (iii) of the lemma is an obvious application of the identity $S(j, x)=$ $B(j, x)$.

In the following paragraphs of this section we will investigate how the tile type condition relates to the classical cotype of a Banach space. For this we will need to be able to view the dyadic intervals inside $[0,1)$ as the time intervals of bitiles of a suitable good collection. This is the content of the following lemma.

Lemma 4.4. Let $\mathcal{J}$ be a finite collection of dyadic intervals in $[0,1)$ and define $2^{-N}:=\min _{I \in \mathcal{J}}|I|$.

(i) Let $\mathbf{T}:=\left\{I \times\left[2^{N+1}-2|I|^{-1}, 2^{N+1}\right): I \in \mathcal{J}\right\}$. Then $\mathbf{T}$ is an up-tree and $\mathcal{J}=\left\{I_{P}: P=I_{P} \times \omega_{P} \in \mathbf{T}\right\}$.

(ii) There exists a good collection of up-trees $\mathcal{T}=\left\{\mathbf{T}_{j}\right\}_{j=0}^{N}$ such that $\mathbf{T}=\cup_{j} \mathbf{T}_{j}$ and

$$
\left\{I \in \mathcal{J}:|I|=2^{j-N}\right\}=\left\{I_{P}: P=I_{P} \times \omega_{P} \in \mathbf{T}_{j}\right\}, \quad 0 \leq j \leq N .
$$

(iii) For $1 \leq r<\infty$ and every $f \in L^{r}([0,1) ; X)$ we have the identity

$$
\sum_{j=0}^{N}\left|\sum_{P \in \mathbf{T}_{j}}\left\langle w_{\mathcal{J}}^{\infty} f, w_{P_{d}}\right\rangle w_{P_{d}}(x)\right|^{r}=\sum_{j=0}^{N}\left|\sum_{I \in \mathcal{J},|I|=2^{-j}}\left\langle f, h_{I}\right\rangle h_{I}(x)\right|^{r},
$$

where $w_{\mathcal{J}}^{\infty}$ is a unimodular function that depends only on the collection $\mathcal{J}$.

Proof. For (i) it is enough to notice that the tile $T:=[0,1) \times\left[2^{N+1}-2,2^{N+1}\right.$ ) satisfies $P \leq_{u} T$ for all $P \in \mathbb{T}$. We now show (ii). For $I \in \mathcal{J}$ we set $\omega_{I}:=$ $\left[2^{N+1}-2|I|^{-1}, 2^{N+1}\right)$ and for all $0 \leq j \leq N$ we define the trees

$$
\mathbf{T}_{j}:=\left\{P=I \times \omega_{I}: I \in \mathcal{J},|I|=2^{j-N}\right\}
$$

We define an appropriate top $T_{j}$ for each tree $\mathbf{T}_{j}$ by setting

$$
T_{j}:=[0,2) \times\left[2^{N+1}-2^{N-j}, 2^{N+1}-2^{N-j}+1\right) .
$$


Suppose that $P=I_{P} \times \omega_{P} \in \mathbf{T}_{j}$ for some $j \in\{0,1, \ldots, N\}$. Then

$$
\omega_{T_{j}}=\left[2^{N+1}-\left|I_{P}\right|^{-1}, 2^{N+1}-\left|I_{P}\right|^{-1}+1\right) \subset \omega_{P_{u}}
$$

and obviously we always have that $I_{P} \subset I_{T_{k}}$. Thus each $T_{j}$ is a top of $\mathbf{T}_{j}$ and hence each $\mathbf{T}_{j}$ is an up-tree. By construction the sequence $\left\{c\left(\omega_{T_{j}}\right)\right\}_{j \leq N}$ is strictly increasing and furthermore the intervals $\omega_{T_{j}}$ are disjoint. We first show that the collection $\left\{\mathbf{T}_{j}\right\}_{j \leq N}$ satisfies

$$
\mathbf{T}_{j}=\left\{P \in \mathbf{P}: P \leq_{u} T_{j} \text { and } P \not \leq_{u} T_{k} \text { for all } k<j\right\},
$$

where $\mathbf{P}$ is the collection of all the bitiles contained in the trees $\mathbf{T}_{j}$. Let $P \in \mathbf{T}_{j}$. We already saw that $P \leq{ }_{u} T_{j}$. Furthermore for $k<j$ we have that $\sup \omega_{T_{k}} \leq \inf \omega_{T_{j}}$. Observe however that inf $\omega_{T_{j}}=2^{N+1}-2^{N-j}=2^{N+1}-\left|I_{P}\right|^{-1}=\omega_{P_{u}}$ since $P \in \mathbf{T}_{j}$. Thus $\sup \omega_{T_{k}} \leq \inf \omega_{P_{u}}$ which implies that $P \Varangle_{u} T_{k}$ whenever $k<j$. This proves

$$
\mathbf{T}_{j} \subseteq\left\{P \in \mathbf{P}: P \leq_{u} T_{j} \text { and } P \not \leq_{u} T_{k} \text { for all } k<j\right\} .
$$

Now assume that $P \in\left\{P \in \mathbf{P}: P \leq_{u} T_{j}\right.$ and $P \leq_{u} T_{k}$ for all $\left.k<j\right\}$. Then we have $P \leq_{u} T_{j}$ thus $\omega_{T_{j, u}} \subseteq \omega_{P_{u}}$ which implies that $2^{N+1}-\left|I_{P}\right|^{-1} \leq 2^{N+1}-2^{N-j} \Leftrightarrow$ $\left|I_{P}\right| \leq 2^{j-N}$. We claim that in fact $\left|I_{P}\right|=2^{j-N}$. Indeed, if $\left|I_{P}\right| \leq 2^{(j-1)-N}$ then we would get that $\omega_{T_{j-1, u}} \subseteq \omega_{P_{u}}$ and this in turn would give that $P \leq_{u} T_{j-1}$ which is a contradiction. Since $P \in \mathbf{P}$ and $\left|I_{P}\right|=2^{j-N}$ we get that $P \in \mathbf{T}_{j}$.

Observe that if $P \in \mathbf{T}_{j}$ for some $j$ then by construction $P \cap T_{k}=\emptyset$ for all $k<j-1$. Thus we have $P \not \mathbb{L}_{u} T_{k} \Leftrightarrow P \not \leq T_{k}$ for $k<j-1$. We now split the collection $\mathcal{T}$ into two collections by setting say $\mathcal{T}_{1}=\left\{\mathbf{T}_{2 j}\right\}_{j}$ and $\mathcal{T}_{2}=\left\{T_{2 j+1}\right\}_{j}$ and each collection $\mathcal{T}_{\nu}, \nu=1,2$, is good. This shows that the original collection $\mathcal{T}$ is a 2 -good collection.

For (iii), remember that the trees $\mathbf{T}_{j}$ share a common top $T=[0,1) \times\left[2^{N+1}-2\right.$, $\left.2^{N+1}\right)$. Thus, Lemma 3.2 implies that

$$
\left|\sum_{P \in \mathbf{T}_{j}}\left\langle f, w_{P_{d}}\right\rangle w_{P_{d}}(x)\right|=\left|\sum_{P \in \mathbf{T}_{j}}\left\langle f w_{T_{u}}^{\infty}, h_{I_{P}}\right\rangle w_{T_{u}}^{\infty} h_{I_{P}}\right|=\left|\sum_{I \in \mathcal{J}}\left\langle f w_{T_{u}}^{\infty}, h_{I_{P}}\right\rangle h_{I_{P}}\right|
$$

which proves the claim in (iii) by setting $w_{\mathcal{J}}^{\infty}:=w_{T_{u}}^{\infty}$ and replacing $f$ by $f w_{\mathcal{J}}^{\infty}$.

Finally we recall the main result proved in [13] concerning the tile type of an interpolation space $X$. Observe that by Lemma 4.3 the down-parts of all bitiles in a good collection $\mathcal{T}$ are disjoint; thus the following proposition is identical to Proposition 3.1 in [13].

Proposition 4.5. For $X$ to have tile type $q$ it is necessary that $X$ be a UMD space and that $q \geq 2$. If a UMD space has tile type $q$, it has tile type $p$ for all $p \in[q, \infty)$. Every Hilbert space has tile type 2, and every complex interpolation space $[Y, H]_{\theta}$, $\theta \in(0,1)$, for a UMD space $Y$ and a Hilbert space $H$ has tile type $2 / \theta$. 


\subsection{Tile type implies cotype}

We observe in this paragraph that the hypothesis that a Banach space $X$ has tile type $q$ implies that the space $X$ has Rademacher and martingale cotype equal to $q$. We recall the relevant definitions.

Let $2 \leq q \leq \infty$. We say that a Banach space $X$ has (Rademacher) cotype $q$ if

$$
\left(\sum_{j \geq 0}\left|x_{j}\right|^{q}\right)^{1 / q} \lesssim\left\|\sum_{j \geq 0} r_{j} x_{j}\right\|_{L^{q}([0,1) ; X)} .
$$

holds uniformly for all finite sequences $\left\{x_{j}\right\}_{j} \subset X$, where $\left\{r_{j}\right\}_{j}$ are the Rademacher functions on $[0,1)$.

On the other hand, we say that $X$ has martingale cotype $q \in[2, \infty]$ (or $M$ cotype $q$ ) if for all $X$-valued martingales $\left\{M_{n}\right\}_{n}$ we have

$$
\left(\mathbb{E} \sum_{n \geq 0}\left|M_{n}-M_{n-1}\right|^{q}\right)^{1 / q} \lesssim\left(\sup _{n} \mathbb{E}\left|M_{n}\right|^{q}\right)^{1 / q} .
$$

Every Banach space trivially has cotype and $M$-cotype $\infty$. In general the notion of $M$-cotype is stronger than that of cotype in the usual sense but the two notions are equivalent in the case that $X$ has the UMD property. Finally we note that martingale cotype is equivalent to Haar cotype, meaning that it is suffices to consider Haar martingales in the definition of $M$-cotype. See [22].

The following proposition shows that tile type implies $M$-cotype with the same index.

Proposition 4.6. Suppose that the Banach space $X$ has tile type $q \geq 2$. Then $X$ is UMD and has $M$-cotype $q$.

Proof. The fact that tile type $q$ implies the UMD property is already contained in Proposition 4.5 but we include a proof here for the sake of completeness. It will suffice to show that

$$
\left\|\sum_{I \in \mathcal{J}} \epsilon_{I}\left\langle f, h_{I}\right\rangle h_{I}\right\|_{L^{r}([0,1) ; X)} \lesssim\|f\|_{L^{r}([0,1) ; X)},
$$

where $\mathcal{J}$ is any finite collection of dyadic intervals inside $[0,1)$,

$f \in L^{r}([0,1) ; X), \epsilon_{I} \in\{-1,+1\}$ and $r$ is some fixed exponent in $(1, \infty)$. Because of the trivial estimate

$$
\begin{aligned}
\left|\sum_{I \in \mathcal{J}} \epsilon_{I}\left\langle f, h_{I}\right\rangle h_{I}\right| & =\left|\sum_{I \in \mathcal{J}, \epsilon_{I}=1}\left\langle f, h_{I}\right\rangle h_{I}-\sum_{I \in \mathcal{J}, \epsilon_{I}=-1}\left\langle f, h_{I}\right\rangle h_{I}\right| \\
& \leq\left|\sum_{I \in \mathcal{J}, \epsilon_{I}=1}\left\langle f, h_{I}\right\rangle h_{I}\right|+\left|\sum_{I \in \mathcal{J}, \epsilon_{I}=-1}\left\langle f, h_{I}\right\rangle h_{I}\right|,
\end{aligned}
$$

it will actually suffice to prove that

$$
\left\|\sum_{I \in \mathcal{J}} \epsilon_{I}\left\langle f, h_{I}\right\rangle h_{I}\right\|_{L^{r}([0,1) ; X)} \lesssim\|f\|_{L^{r}([0,1) ; X)}
$$


whenever $\epsilon_{I} \in\{0,1\}$. However this amounts to showing that

$$
\left\|\sum_{I \in \mathcal{J}^{\prime}}\left\langle f, h_{I}\right\rangle h_{I}\right\|_{L^{r}([0,1) ; X)} \lesssim\|f\|_{L^{r}([0,1) ; X)},
$$

for any finite collection $\mathcal{J}^{\prime}$ of dyadic intervals in $[0,1)$. Consider the up-tree given by (i) of Lemma 4.4 applied to the collection $\mathcal{J}^{\prime}$,

$$
\mathbf{T}:=\left\{I \times\left[2^{N+1}-2|I|^{-1}, 2^{N+1}\right): I \in \mathcal{J}^{\prime}\right\}
$$

where $N$ is such that $|I| \geq 2^{-N}$ for all $I \in \mathcal{J}^{\prime}$. Setting $g w_{T_{u}}^{\infty}:=f$ we use Lemma 3.2 to write

$$
\begin{aligned}
\left\|\sum_{I \in \mathcal{J}^{\prime}}\left\langle f, h_{I}\right\rangle h_{I}\right\|_{L^{q}([0,1) ; X)}^{q} & =\int\left|\sum_{P \in \mathbf{T}}\left\langle f, h_{I_{P}}\right\rangle h_{I_{P}}(x)\right|^{q} d x \\
& =\int\left|\sum_{P \in \mathbf{T}}\left\langle g, w_{P_{d}}\right\rangle w_{P_{d}}(x)\right|^{q} d x \lesssim\|f\|_{L^{q}([0,1) ; X)}^{q}
\end{aligned}
$$

where in the last inequality we used the tile type hypothesis for the collection consisting of the single tree $\mathbf{T}$. This however is the UMD condition for Haar martingales with $r=q$.

We will now show that $X$ has martingale cotype $q$. By Lemma 4.4 we have for every positive integer $N$ that

$$
\sum_{j=0}^{N}\left|\sum_{|I|=2^{-k}}\left\langle f, h_{I}\right\rangle h_{I}(x)\right|^{q}=\sum_{j=0}^{N}\left|\sum_{P \in \mathbf{T}_{j}}\left\langle w_{N}^{\infty} f, w_{P_{d}}\right\rangle w_{P_{d}}(x)\right|^{q},
$$

where $\left\{\mathbf{T}_{j}\right\}_{j=0}^{N}$ is a good collection of up-trees. Since $X$ has tile type $q$ the right hand side is controlled by $\|f\|_{L^{q}([0,1) ; X)}$. Thus

$$
\sum_{0 \leq k \leq N}\left\|\sum_{|I|=2^{-k}}\left\langle f, h_{I_{P}}\right\rangle h_{I_{P}}\right\|_{L^{q}([0,1) ; X)}^{q} \lesssim\|f\|_{L^{q}([0,1) ; X)}^{q},
$$

with the implicit constant not depending on $N$. This is the cotype condition for Haar martingale differences which by [22] is equivalent to $X$ having martingale cotype $q$.

\subsection{Vector-valued Lépingle inequality}

The variational Carleson theorem, in the scalar case, depends on certain jump inequalities originally due to Lépingle in [17]. This fact has been recorded and well understood in several papers, for example in [11], [20], and [15]. For the Banach space case that we are considering we will need the appropriate vector-valued extension proved by Pisier and $\mathrm{Xu}$ in [23]: 
Theorem 4.7 (Theorem 4.3 in [23]). Suppose that $X$ has cotype $\tau$ for all $\tau>q$. Then we have Lépingle's inequality for functions $f \in L^{p}\left(\mathbb{R}_{+} ; X\right)$

$$
\left\|\mathbb{E}_{n} f\right\|_{L^{p}\left(\mathbb{R}_{+} ; \mathcal{V}^{r}(X)\right)} \lesssim\|f\|_{L^{p}\left(\mathbb{R}_{+} ; X\right)},
$$

for all $r>q$ and $1<p<\infty$.

Here we remember that $\mathbb{E}_{n}$ is the conditional expectation with respect to a dyadic interval of length $2^{n}$, as defined in (2.1). By Proposition 4.6 one can replace the cotype $\tau>q$ hypothesis in Theorem 4.7 by the hypothesis that $X$ has tile type $\tau$ for all $\tau>q$. We will use this fact in what follows without further comment.

\section{Linearization of the variational Carleson operator}

In this section we linearize the variational norm of the partial Walsh-Fourier sums of a function $f$, using more or less standard arguments as in [28], [13], [11], and [20]. We reduce the statement of Theorem 1.3 to an analogous statement about some closely related linearized versions of the variational Carleson operator which are more amenable to time-frequency analysis techniques and interpolation. We assume the tile type hypothesis throughout the section in the statements of our reduced theorems.

For any collection of bitiles $\mathbf{P}$ we define the operator

$$
C_{r, \mathbf{P}} f(x):=\sup _{K, N_{0}<\cdots<N_{K}}\left(\sum_{j=1}^{K}\left|\sum_{P \in \mathbf{P}}\left\langle f, w_{P_{d}}\right\rangle w_{P_{d}}(x) \mathbf{1}_{\left\{N_{j} \in\left[\omega_{P_{u}}\right), N_{j-1} \notin \dot{\omega}_{P}\right\}}\right|^{r}\right)^{1 / r}
$$

where the supremum is taken over all positive integers $K$ and all nonnegative real numbers $N_{0}<N_{1}<\cdots<N_{K}$. There is a symmetric version, denoted by $\tilde{C}_{r, \mathbf{P}} f(x)$, in which the down-tiles are replaced by up-tiles and the condition in the indicator is replaced by $N_{j} \notin \stackrel{\circ}{\omega}_{P}, N_{j-1} \in\left(\omega_{P_{d}}\right]$, namely:

$$
\tilde{C}_{r, \mathbf{P}} f(x):=\sup _{K, N_{0}<\cdots<N_{K}}\left(\sum_{j=1}^{K}\left|\sum_{P \in \mathbf{P}}\left\langle f, w_{P_{u}}\right\rangle w_{P_{u}}(x) \mathbf{1}_{\left\{N_{j} \in\left(\omega_{P_{d}}\right], N_{j-1} \notin \dot{\omega}_{P}\right\}}\right|^{r}\right)^{1 / r} .
$$

These operators are formed over all bitiles, namely, one only requires $I_{P} \subset[0, \infty)$.

The second statement in Theorem 1.1 will be a consequence of the following theorem for $C_{r, \mathbf{P}}$ and its symmetric analog for $\tilde{C}_{r, \mathbf{P}}$.

Theorem 5.2. Let $X$ be a Banach space with tile type $\tau$ for all $\tau>q$ and let $\mathbf{P}$ be any collection of bitiles. We have

$$
\left\|C_{r, \mathbf{P}} f\right\|_{L^{p}\left(\mathbb{R}_{+} ; X\right)} \lesssim_{p, r, q}\|f\|_{L^{p}\left(\mathbb{R}_{+} ; X\right)},
$$

whenever $q<r<\infty$ and $0<1 / p<1 / r^{\prime}-(q-2) / r$. 
Concerning the proof of this theorem, we focus on the operator $C_{r, \mathbf{P}} f(x)$, using in particular the partial order on bitiles and their organization into trees, among other techniques. The main hypothesis is that the space $X$ has finite tile type arbitrarily close to some number $q$. The reader here should think of the tile type hypothesis in the formulation given for families of up-trees, that is, in the equivalent formulation of the $u$-tile type. For the operator, $\tilde{C}_{r, \mathbf{P}} f$, the proof is completely symmetric, in view of Lemma 4.1, where the role of the down tile $P_{d}$ is analogous to that of the up-tile, and vice-versa. For all the considerations concerning the operator $\tilde{C}_{r, \mathbf{P}} f$ we switch our point of view to the formulation of the $d$-tile type. Bearing this in mind it is routine to adjust the arguments in this paper, given for the operator $C_{r, \mathbf{P}} f$, in order to give the corresponding proof for the symmetric operator $\tilde{C}_{r, \mathbf{P}} f$. We thus omit any further discussion concerning the proof of Theorem 5.2 for the dual operator $\tilde{C}_{r, \mathbf{P}} f$.

We briefly describe how to deduce Theorem 1.3 from Theorem 5.2.

Proof of Theorem 1.3. For integers $0<\zeta<\zeta^{\prime}$, let $\Omega_{\zeta, \zeta^{\prime}}$ be the maximal dyadic intervals $\omega \subset\left[\zeta, \zeta^{\prime}\right)$. These intervals partition $\left[\zeta, \zeta^{\prime}\right)$, and moreover we have

$$
S_{\zeta^{\prime}} f-S_{\zeta} f=\sum_{\substack{P \text { is a tile } \\ I_{P} \subset[0,1), \omega_{P} \in \Omega_{\zeta, \zeta^{\prime}}}}\left\langle f, w_{P}\right\rangle w_{P} .
$$

This follows from Corollary 8.3 in [28] and is a variant of the formula on p. 68-69 of [28]. Now, let $\Omega_{\zeta, \zeta^{\prime}}^{u / d}$ be comprised of those intervals $\omega \in \Omega_{\zeta, \zeta^{\prime}}$ for which $\omega$ is the up/down-half of its parent. Let $\mathbf{P}_{\zeta, \zeta^{\prime}}^{u / d}$ be the collection of bitiles such that $\omega_{P_{u / d}} \in \Omega_{\zeta, \zeta^{\prime}}^{u / d}$, and $I_{P} \subset[0,1)$. We then have

$$
S_{\zeta^{\prime}} f-S_{\zeta} f=\sum_{\sigma \in\{u, d\}} \sum_{P \in \mathbf{P}_{\zeta, \zeta^{\prime}}^{\sigma}}\left\langle f, w_{P_{\sigma}}\right\rangle w_{P_{\sigma}} .
$$

We have $P \in \mathbf{P}_{\zeta, \zeta^{\prime}}^{d}$ if and only if $I_{P} \subset[0,1), \zeta \notin \stackrel{\circ}{\omega}_{P}$, and $\zeta^{\prime} \in\left[\omega_{P_{u}}\right)$, conditions in agreement with the conditions on $N_{j-1}$ and $N_{j}$ in the definition of $C_{r, \mathbf{P}} f$. In the symmetric case, we have $P \in \mathbf{P}_{\zeta, \zeta^{\prime}}^{u}$ if and only if $I_{P} \subset[0,1), \zeta \in\left(\omega_{P_{d}}\right]$, and $\zeta^{\prime} \notin \stackrel{\circ}{\omega}_{P}$. All together, for any $K, N_{0}<\cdots<N_{K}$, we have

$$
\begin{aligned}
& \sum_{j=1}^{K}\left|S_{N_{j-1}} f-S_{N_{j}}\right|^{r}=\sum_{j=1}^{K}\left|\sum_{\sigma \in\{u, d\}} \sum_{P \in \mathbf{P}_{N_{j-1}, N_{j}}^{\sigma}}\left\langle f, w_{P_{\sigma}}\right\rangle w_{P_{\sigma}}\right|^{r} \\
& \lesssim \sum_{\sigma \in\{u, d\}} \sum_{j=1}^{K}\left|\sum_{P \in \mathbf{P}_{N_{j-1}, N_{j}}^{\sigma}}\left\langle f, w_{P_{\sigma}}\right\rangle w_{P_{\sigma}}\right|^{r} \lesssim\left(C_{r, \mathbf{P}_{1}} f\right)^{r}+\left(\tilde{C}_{r, \mathbf{P}_{2}} f\right)^{r},
\end{aligned}
$$

for some fixed collections of bitiles $\mathbf{P}_{1}$ and $\mathbf{P}_{2}$. Using Theorem 5.2, which is valid for arbitrary collections $\mathbf{P}$, and the pointwise inequality just proved, completes the proof. 
The first statement in Theorem 1.1 is the content of:

Proposition 5.4. Let $X$ be some Banach space and suppose that for any collection of bitiles $\mathbf{P}$, the operator $C_{r, \mathbf{P}}$, or the operator $\tilde{C}_{r, \mathbf{P}}$, satisfies the conclusion of Theorem 5.2 with $p=r$,

$$
\left\|C_{r, \mathbf{P}} f\right\|_{L^{r}\left(\mathbb{R}_{+} ; X\right)} \lesssim\|f\|_{L^{r}\left(\mathbb{R}_{+} ; X\right)},
$$

whenever $q<r<\infty$. Then $X$ has tile type $\tau$ for all $\tau>q$ and, a fortiori, $X$ has cotype $\tau$ for all $\tau>q$.

Proof. We will prove the proposition assuming that the operator $C_{r, \mathbf{P}}$ is bounded on $L^{r}\left(\mathbb{R}_{+} ; X\right)$ for all $r>q$. Let $\mathcal{T}=\left\{\mathbf{T}_{j}\right\}$ be a u-good collection of up-trees. By (ii) and (iii) of Lemma 4.3 there is an increasing sequence of integer valued functions $\left\{N_{j}(x)\right\}_{j}$, such that

$$
\begin{gathered}
\sum_{j}\left\|\sum_{P \in \mathbf{T}_{j}}\left\langle f, w_{P_{d}}\right\rangle w_{P_{d}}\right\|_{L^{r}\left(\mathbb{R}_{+} ; X\right)}^{r} \\
=\sum_{j}\left\|\sum_{P \in \mathbf{P}}\left\langle f, w_{P_{d}}\right\rangle w_{P_{d}} \mathbf{1}_{\left\{N_{j} \in\left[\omega_{P_{u}}\right), N_{j-1} \notin \dot{\omega}_{P}\right\}}\right\|_{L^{r}\left(\mathbb{R}_{+} ; X\right)}^{r} \\
\leq\left\|C_{r, \mathbf{P}} f\right\|_{L^{r}\left(\mathbb{R}_{+} ; X\right)}^{r} \lesssim_{r}\|f\|_{L^{r}\left(\mathbb{R}_{+} ; X\right)}^{r},
\end{gathered}
$$

since $C_{r, \mathbf{P}}$ is bounded on $L^{r}\left(\mathbb{R}_{+} ; X\right)$. If the hypothesis is true for $\tilde{C}_{r, \mathbf{P}}$ we consider $d$-good collections of trees and show the corresponding statement for the $d$-tile type. The conclusion then follows by using the analogue of Lemma 4.3 for $d$-good collections.

Following [11] we consider the linearized version of $C_{r, \mathbf{P}}$ given by

$$
C_{\mathbf{P}} f(x)=C_{r, a, \mathbf{P}} f(x):=\sum_{j=1}^{K(x)} \sum_{P \in \mathbf{P}}\left\langle f, w_{P_{d}}\right\rangle w_{P_{d}}(x) \mathbf{1}_{\left\{N_{j}(x) \in\left[\omega_{P_{u}}\right), N_{j-1}(x) \notin \dot{\omega}_{P}\right\}} a_{j}(x),
$$

where $K, N_{1}, \ldots N_{K}: \mathbb{R}_{+} \rightarrow \mathbb{R}_{+}$are arbitrary measurable functions and $a=\left\{a_{j}\right\}_{j}$ is a sequence of $X^{*}$-valued functions with $\sum_{j=1}^{K(x)}\left|a_{j}(x)\right|^{r^{\prime}}=1$. The expression for the operator $C_{\mathbf{P}}$ can be simplified by writing

$$
\begin{aligned}
C_{\mathbf{P}} f(x) & =\sum_{P \in \mathbf{P}}\left\langle f, w_{P_{d}}\right\rangle w_{P_{d}}(x) \sum_{j=1}^{K(x)} \mathbf{1}_{\left\{N_{j}(x) \in\left[\omega_{P_{u}}\right), N_{j-1}(x) \notin \grave{\omega}_{P}\right\}} a_{j}(x) \\
& =\sum_{P \in \mathbf{P}}\left\langle f, w_{P_{d}}\right\rangle w_{P_{d}}(x) a_{P}(x),
\end{aligned}
$$

where

$$
a_{P}(x):=\sum_{j=1}^{K(x)} \mathbf{1}_{\left\{N_{j}(x) \in\left[\omega_{P_{u}}\right), N_{j-1}(x) \notin \dot{\omega}_{P}\right\}} a_{j}(x)
$$


The operator $C_{\mathbf{P}}$ depends on both $r$ and the choice of the sequence $a$, but we suppress this fact in what follows in order to simplify our notation.

Note here that our assumption that $X$ has tile type $\tau \in(q, \infty)$ can be replaced by the assumption that $X$ has tile type exactly $q$. This is because all our conclusions are given in terms of open intervals with respect to $p, r$, and $q$. Via a standard restricted weak-type interpolation argument, as for example in Chapter 3 of [28], the proof of Theorem 5.2 reduces to the proof of the following statement.

Proposition 5.6. Suppose that $X$ is a Banach space with tile type $q \geq 2$. Let $F, E \subset \mathbb{R}_{+}$be measurable sets with $|F|,|E|<+\infty$. Then there are major subsets $E^{\prime} \subseteq E$ and $F^{\prime} \subseteq F$ with either $E^{\prime}=E$ or $F^{\prime}=F$, such that, for all $f: X \rightarrow \mathbb{R}_{+}$ with $|f| \leq \mathbf{1}_{F^{\prime}}$, and all $g: X^{*} \rightarrow \mathbb{R}_{+}$with $|g| \leq \mathbf{1}_{E^{\prime}}$, we have

$$
\left|\left\langle C_{\mathbf{P}} f, g\right\rangle\right| \lesssim|F|^{1 / p}|E|^{1 / p^{\prime}}
$$

whenever $\max \left(q, p^{\prime}(q-1)\right)<r<\infty$.

Here we say that $E^{\prime} \subset E$ is a major subset of $E$ if $\left|E^{\prime}\right| \geq \frac{1}{2}|E|$.

Remark 5.7. Observe that

$$
\left|\left\langle C_{\mathbf{P}} f, g\right\rangle\right| \leq \sum_{P \in \mathbf{P}}\left|\left\langle f, w_{P_{d}}\right\rangle\left\langle w_{P_{d}} a_{P}, g\right\rangle\right|=\sum_{P \in \mathbf{P}} \epsilon_{P}\left\langle f, w_{P_{d}}\right\rangle\left\langle w_{P_{d}} a_{P}, g\right\rangle=\left|\left\langle C_{\mathbf{P}}^{+} f, g\right\rangle\right|
$$

for some choice of signs $\epsilon_{P} \in\{-1,+1\}$, where

$$
C_{\mathbf{P}}^{+} f(x):=\sum_{P \in \mathbf{P}} \epsilon_{P}\left\langle f, w_{P_{d}}\right\rangle w_{P_{d}}(x) a_{P}(x) .
$$

We will thus prove the estimate in Proposition 5.6 for the larger operator $C_{\mathbf{P}}^{+}$, which we rename $C_{\mathbf{P}}$, and require that $\mathbf{P}$ be any finite collection of bitiles $\mathbf{P}$.

\section{The tree lemma}

Let $\mathbf{P}$ be a finite collection of bitiles. The density of the collection $\mathbf{P}$ is

$$
\operatorname{density}(\mathbf{P}):=\sup _{P \in \mathbf{P}} \sup _{P^{\prime} \geq P}\left(\frac{1}{\left|I_{P^{\prime}}\right|} \int_{I_{P^{\prime}}}|g(x)|^{r^{\prime}} \sum_{j: N_{j}(x) \in \omega_{P^{\prime}}}\left|a_{j}(x)\right|^{r^{\prime}} d x\right)^{1 / r^{\prime}}
$$

where we remember that $N_{j}: \mathbb{R}_{+} \rightarrow \mathbb{R}_{+}$are measurable functions, $q \geq 2$ is the tile type of the Banach space $X$, and $r>q$. We define the size of a collection $\mathbf{P}$ to be

$$
\operatorname{size}(\mathbf{P}):=\sup _{\mathbf{T} \subseteq \mathbf{P} \text { up }- \text { tree }}\left(\frac{1}{\left|I_{T}\right|} \int\left|\sum_{P \in \mathbf{T}}\left\langle f, w_{P_{d}}\right\rangle w_{P_{d}}(x)\right|^{q} d x\right)^{1 / q} .
$$


Lemma 6.1 (Tree lemma). For every tree $\mathbf{T}$ we have

$$
\left\|g C_{\mathbf{T}} f\right\|_{L^{s}\left(\mathbb{R}_{+}\right)}=\left\|\sum_{P \in \mathbf{T}}\left\langle f, w_{P_{d}}\right\rangle w_{P_{d}} a_{P} g\right\|_{L^{s}\left(\mathbb{R}_{+}\right)} \lesssim \operatorname{size}(\mathbf{T}) \operatorname{density}(\mathbf{T})\left|I_{T}\right|^{1 / s},
$$

for all $1 \leq s \leq r^{\prime}$.

We will prove the lemma for the case $s=r^{\prime}$ which, by Hölder's inequality, implies the conclusion for $1 \leq s \leq r^{\prime}$ as well. Let $\mathcal{J}$ be the collection of maximal dyadic intervals contained in $I_{T}$ that do not contain any $I_{P}, P \in \mathbf{T}$. The intervals in the collection $\mathcal{J}$ form a partition of $I_{T}$ thus

$$
\begin{aligned}
\left\|g C_{\mathbf{T}} f\right\|_{L^{r^{\prime}\left(\mathbb{R}_{+}\right)}} & =\left(\sum_{J \in \mathcal{J}} \int_{J}\left|\sum_{P \in \mathbf{T}, I_{P} \supsetneq J} \epsilon_{P}\left\langle f, w_{P_{d}}\right\rangle w_{P_{d}}(x) a_{P}(x) g(x)\right|^{r^{\prime}} d x\right)^{1 / r^{\prime}} \\
& =\left(\sum_{J \in \mathcal{J}}\left\|\sum_{P \in \mathbf{T}, I_{P} \supsetneq J} \epsilon_{P}\left\langle f, w_{P_{d}}\right\rangle w_{P_{d}} a_{P} g\right\|_{L^{r^{\prime}(J)}}^{r^{\prime}}\right)^{1 / r^{\prime}} .
\end{aligned}
$$

We set, for $P \in \mathbf{T}$ and $j \geq 1$,

$$
A(P, j):=I_{P} \cap\left\{x: N_{j-1}(x) \notin \stackrel{\circ}{\omega}_{P}, N_{j}(x) \in\left[\omega_{P_{u}}\right)\right\} .
$$

We gather some auxiliary calculations in the following lemma.

Lemma 6.2. Fix a tree $\mathbf{T}$ and a top $T$ of $\mathbf{T}$ and consider the partition of $I_{T}$ into intervals $J \in \mathcal{J}$. Let $J \in \mathcal{J}$ and denote by $J^{(1)}$ the dyadic parent of $J$. There exist bitiles $Q(J) \in \mathbf{T}$ and $P(J)=J^{(1)} \times \omega(J)$ such that:

(i) $Q(J) \leq P(J) \leq T$.

(ii) For every $j \in[1, K(x)]$ we have the pointwise inequality

$$
\mathbf{1}_{J} \mathbf{1}_{A(P, j)} \leq \mathbf{1}_{\left\{x: N_{j}(x) \in \omega(J)\right\}} .
$$

(iii) We have the estimate

$$
\int_{J}|g(x)|^{r^{\prime}} \sum_{j: N_{j}(x) \in \omega(J)}\left|a_{j}(x)\right|^{r^{\prime}} d x \lesssim|J| \operatorname{density}(\mathbf{T})^{r^{\prime}} .
$$

Proof. Fix some $J \in \mathcal{J}$. Since $J$ is maximal among intervals that contain no $I_{P}$, $P \in \mathbf{T}$, there is some bitile $Q(J) \in \mathbf{T}$ such that $I_{Q(J)} \subseteq J^{(1)}$, where $J^{(1)}$ is the dyadic parent of $J$. Observe that we must have $J^{(1)} \subseteq I_{T}$. Define the frequency interval $\omega(J)$ with $|\omega(J)|=2 /\left|J^{(1)}\right|$ and such that $\omega_{T} \subseteq \omega(J) \subseteq \omega_{Q(J)}$. Thus the bitile $P(J):=J^{(1)} \times \omega(J)$ satisfies $Q(J) \leq P(J) \leq T$. This proves (i).

Now for all bitiles $P \in \mathbf{T}$ such that $I_{P} \cap J \neq \emptyset$ we have $J \subsetneq I_{P}$ by the maximality of $J$ which implies that $\left|I_{P}\right| \geq\left|J^{(1)}\right|$. For every such $P$ we thus have $\emptyset \neq \omega_{T} \subseteq \omega_{P} \cap \omega(J)$ and so $\omega_{P} \subseteq \omega(J)$. We conclude

$$
\bigcup_{P \in \mathbf{T}: I_{P} \cap J \neq \emptyset} \omega_{P} \subseteq \omega(J) .
$$

Let $x$ be such that $\mathbf{1}_{J}(x) \mathbf{1}_{A(P, j)}(x) \neq 0$. Then $I_{P} \cap J \neq \emptyset$ and $N_{j}(x) \in \omega_{P_{u}} \subset \omega_{P}$ $\subseteq \omega(J)$. The previous inclusion thus implies $\mathbf{1}_{J} \mathbf{1}_{A(P, j)} \leq \mathbf{1}_{\left\{x: N_{j}(x) \in \omega(J)\right\}}$. 
Finally, we have $P(J) \geq Q(J)$ and $Q(J) \in \mathbf{T}$. Thus

$$
\begin{array}{r}
\int_{J}|g(x)|^{r^{\prime}} \sum_{j: N_{j}(x) \in \omega(J)}\left|a_{j}(x)\right|^{r^{\prime}} d x \lesssim|J| \frac{1}{\left|J^{(1)}\right|} \int_{J^{(1)}}|g(x)|^{r^{\prime}} \sum_{j: N_{j}(x) \in \omega(J)}\left|a_{j}(x)\right|^{r^{\prime}} d x \\
=|J| \frac{1}{\left|I_{P(J)}\right|} \int_{I_{P(J)}}|g(x)|^{r^{\prime}} \sum_{j: N_{j}(x) \in \omega_{P(J)}}\left|a_{j}(x)\right|^{r^{\prime}} d x \leq|J| \operatorname{density}(\mathbf{T})^{r^{\prime}}
\end{array}
$$

by the definition of density.

For $\tau \in\{u=$ up, $d=$ down $\}$ define the functions

$$
\begin{aligned}
F_{J, \tau}(x) & :=\mathbf{1}_{J}(x) g(x) \sum_{P \in \mathbf{T}_{\tau}, I_{P} \supsetneq J} \epsilon_{P}\left\langle f, w_{P_{d}}\right\rangle w_{P_{d}}(x) a_{P}(x) \\
& =\mathbf{1}_{J}(x) g(x) \sum_{P \in \mathbf{T}_{\tau}, I_{P} \supsetneq J} \sum_{j=1}^{K(x)} \epsilon_{P}\left\langle f, w_{P_{d}}\right\rangle w_{P_{d}}(x) a_{j}(x) \mathbf{1}_{A(P, j)}(x) .
\end{aligned}
$$

Since every tree $\mathbf{T}$ can be written as a union of its up-part $\mathbf{T}_{u}$ and its down-part $\mathbf{T}_{d}$, we have the estimate

$$
\left\|g C_{\mathbf{T}} f\right\|_{L^{r^{\prime}\left(\mathbb{R}_{+}\right)}} \leq\left(\sum_{J \in \mathcal{J}}\left\|F_{J, d}\right\|_{L^{r^{\prime}\left(\mathbb{R}_{+}\right)}}^{r^{\prime}}\right)^{1 / r^{\prime}}+\left(\sum_{J \in \mathcal{J}}\left\|F_{J, u}\right\|_{L^{r^{\prime}\left(\mathbb{R}_{+}\right)}}^{r^{\prime}}\right)^{1 / r^{\prime}} .
$$

We estimate the two terms appearing in the previous sum separately.

Lemma 6.3. We have

$$
\left(\sum_{J \in \mathcal{J}}\left\|F_{J, d}\right\|_{L^{r^{\prime}}\left(\mathbb{R}_{+}\right)}^{r^{\prime}}\right)^{1 / r^{\prime}} \lesssim\left|I_{T}\right|^{1 / r^{\prime}} \operatorname{density}(\mathbf{T}) \operatorname{size}(\mathbf{T})
$$

Proof. The function $F_{J, d}$ can be written as

$$
F_{J, d}(x)=\mathbf{1}_{J}(x) g(x) \sum_{P \in \mathbf{T}_{d}, I_{P} \supsetneq J} \sum_{j=1}^{\infty} \epsilon_{P}\left\langle f, w_{P_{d}}\right\rangle w_{P_{d}}(x) a_{j}(x) \mathbf{1}_{[j, \infty)}(K(x)) \mathbf{1}_{A(P, j)}(x) .
$$

Now consider two pairs $(j, P) \neq\left(j^{\prime}, P^{\prime}\right)$ appearing in the previous sum, with $j, j^{\prime} \geq 1$ and $P, P^{\prime} \in \mathbf{T}_{d}$ such that $I_{P}, I_{P^{\prime}} \supsetneq J$. We claim that $A(P, j) \cap A\left(P^{\prime}, j^{\prime}\right)=\emptyset$. Indeed, since $\mathbf{T}_{d}$ is a down-tree we have $\omega_{T_{d}} \subseteq \omega_{P_{d}} \cap \omega_{P_{d}^{\prime}} \neq \emptyset$ and thus the bigger interval contains the smaller. For example we have $\omega_{P_{d}^{\prime}} \subseteq \omega_{P_{d}} \Rightarrow \omega_{P^{\prime}} \subseteq \omega_{P}$.

If $j^{\prime}<j \Leftrightarrow j^{\prime} \leq j-1$ and $x \in A(P, j)$ then we have $\left(x, N_{j}(x)\right) \in P_{u}$ and $\left(x, N_{j-1}(x)\right) \notin \stackrel{\circ}{P}$. Since $N_{j}(x)>N_{j-1}(x)$ we get that necessarily $N_{j-1}(x) \leq$ $\min \omega_{P}$. Thus

$$
N_{j^{\prime}}(x) \leq N_{j-1}(x) \leq \min \omega_{P} \leq \min \omega_{P^{\prime}} \Longrightarrow N_{j^{\prime}}(x) \notin \omega_{P_{u}^{\prime}} \Longrightarrow x \notin A\left(P^{\prime}, j^{\prime}\right) .
$$


Suppose now that $j^{\prime}>j \Leftrightarrow j \leq j^{\prime}-1$. If $x \in A\left(P^{\prime}, j^{\prime}\right)$ then $\left(x, N_{j^{\prime}}(x)\right) \in P_{u}^{\prime}$ and $\left(x, N_{j^{\prime}-1}(x)\right) \notin \stackrel{\circ}{P^{\prime}}$. Since $N_{j^{\prime}}(x) \geq N_{j^{\prime}-1}(x)$ we must have $N_{j^{\prime}-1}(x) \leq \min \omega_{P^{\prime}}$. Thus

$$
N_{j}(x) \leq N_{j^{\prime}-1}(x) \leq \min \omega_{P^{\prime}}=\min \omega_{P_{d}^{\prime}}<\min \omega_{P_{u}}
$$

since $\omega_{P_{d}^{\prime}} \subseteq \omega_{P_{d}}$. Thus $N_{j}(x) \notin \omega_{P_{u}}$ which implies that $x \notin A(P, j)$. In every case we get that $A(P, j) \cap A\left(P^{\prime}, j^{\prime}\right) \neq \emptyset \Rightarrow j=j^{\prime}$. However all the up-parts $P_{u}, P \in \mathbf{T}_{d}$, are disjoint since $\mathbf{T}_{d}$ is a down-tree, so we cannot have $N_{j}(x) \in \omega_{P_{u}} \cap \omega_{P_{u}^{\prime}}$ with $P \neq P^{\prime}$. We conclude that $A(P, j) \cap A\left(P^{\prime}, j^{\prime}\right) \neq \emptyset \Rightarrow(P, j)=\left(P^{\prime}, j^{\prime}\right)$, as claimed.

The disjointness property of the $A(P, j)$ implies that

$$
\left|F_{J, d}(x)\right| \leq|g(x)| \sup _{\substack{P \in \mathbf{T}_{d} \\ I_{P} \supsetneq J}} \sup _{1 \leq j \leq K(x)} \frac{\left|\left\langle f, w_{P_{d}}\right\rangle\right|}{\left|I_{P}\right|^{1 / 2}}\left|a_{j}(x)\right| \mathbf{1}_{J}(x) \mathbf{1}_{A(P, j)}(x)
$$

Furthermore, by the definition of size it is not hard to see that $\left.\left|\left\langle f, w_{P_{d}}\right\rangle /\right| I_{P}\right|^{1 / 2} \leq$ size(T), by testing the definition against a tree consisting of a single bitile $P$. Combining this estimate with (ii) of Lemma 6.2 and (6.4) we get that, for any fixed $x$,

$$
\begin{aligned}
\left|F_{J, d}(x)\right| & \leq \operatorname{size}(\mathbf{T})|g(x)| \sup _{1 \leq j \leq K(x)}\left|a_{j}(x)\right| \mathbf{1}_{\left\{y: N_{j}(y) \in \omega(J)\right\}}(x) \\
& \leq \operatorname{size}(\mathbf{T})|g(x)|\left(\sum_{j: N_{j}(x) \in \omega(J)}\left|a_{j}(x)\right|^{r^{\prime}}\right)^{1 / r^{\prime}} .
\end{aligned}
$$

Integrating the previous estimate raised to the power $r^{\prime}$ yields

$$
\begin{aligned}
\left\|F_{d, J}\right\|_{L^{r^{\prime}\left(\mathbb{R}_{+}\right)}}^{r^{\prime}} & \lesssim \operatorname{size}(\mathbf{T})^{r^{\prime}} \int_{J}|g(x)|^{r^{\prime}} \sum_{j: N_{j}(x) \in \omega(J)}\left|a_{j}(x)\right|^{r^{\prime}} d x \\
& \lesssim|J| \operatorname{size}(\mathbf{T})^{r^{\prime}} \operatorname{density}(\mathbf{T})^{r^{\prime}},
\end{aligned}
$$

by Lemma 6.2 , (iii). Since the collection $\mathcal{J}$ partitions $I_{T}$, summing in $J \in \mathcal{J}$ gives

$$
\begin{aligned}
\left(\sum_{J \in \mathcal{J}}\left\|F_{d, J}\right\|_{L^{r^{\prime}\left(\mathbb{R}_{+}\right)}}^{r^{\prime}}\right)^{1 / r^{\prime}} & \lesssim \operatorname{size}(\mathbf{T}) \operatorname{density}(\mathbf{T})\left(\sum_{J \in \mathcal{J}}|J|\right)^{1 / r^{\prime}} \\
& =\left|I_{T}\right|^{1 / r^{\prime}} \operatorname{size}(\mathbf{T}) \operatorname{density}(\mathbf{T})
\end{aligned}
$$

as we wanted.

The proof for the up-part is more involved.

Lemma 6.5. We have

$$
\left(\sum_{J \in \mathcal{J}}\left\|F_{J, u}\right\|_{L^{r^{\prime}\left(\mathbb{R}_{+}\right)}}^{r^{\prime}}\right)^{1 / r^{\prime}} \lesssim\left|I_{T}\right|^{1 / r^{\prime}} \operatorname{density}(\mathbf{T}) \operatorname{size}(\mathbf{T})
$$


Proof. We fix some $J \in \mathcal{J}$ and $x \in J$ so that $F_{J, u}(x) \neq 0$. We use Lemma 3.2 in order to write the function $F_{J, u}$ in the form

$$
\begin{array}{r}
F_{J, u}(x)=\mathbf{1}_{J}(x) g(x) w_{T_{u}}^{\infty}(x) \\
\times \sum_{\substack{1 \leq j \leq K(x) \\
j: N_{j}(x) \in \omega(J)}} \sum_{P \in \mathbf{T}_{u}, I_{P} \supsetneq J} \epsilon_{P} \epsilon_{P T}\left\langle f, w_{P_{d}}\right\rangle h_{I_{P}}(x) a_{j}(x) \mathbf{1}_{A(P, j)}(x),
\end{array}
$$

where $\epsilon_{P} \epsilon_{P T} \in\{-1,+1\}$ and $w_{T_{u}}^{\infty}$ is unimodular and depends only on the upper tile of the top $T$ of $\mathbf{T}$. Now for every $1 \leq j \leq K(x)$ with $N_{j}(x) \in \omega(J)$, consider the inner sum

$$
\sum_{P \in \mathbf{T}_{u}, I_{P} \supsetneq J} \epsilon_{P} \epsilon_{P T}\left\langle f, w_{P_{d}}\right\rangle h_{I_{P}}(x) a_{j}(x) \mathbf{1}_{A(P, j)}(x) .
$$

Consider $j$ and $x$ such that $\mathbf{1}_{A(P, j)}(x) \neq 0$ in (6.6) and examine which bitiles $P \in \mathbf{T}_{u}$ contribute to it. For such bitiles we must have $N_{j-1}(x) \notin \omega_{P}$ and $N_{j}(x) \in$ $\omega_{P_{u}}$. Now the frequency intervals $\omega_{P_{u}}, P \in \mathbf{T}_{u}$, all contain the top interval $\omega_{T_{u}}$ and thus they are nested. This nestedness property implies that if $N_{j}(x) \in \omega_{P_{u}^{\prime}}$ is satisfied for some $P^{\prime} \in \mathbf{T}_{u}$ then it will also be satisfied for all $P \in \mathbf{T}_{u}$ with $\omega_{P_{u}} \supset \omega_{P_{u}^{\prime}}$. Likewise, all the $\omega_{P}$ of bitiles $P \in \mathbf{T}_{u}$ that contribute to the sum are nested since they all contain the top interval $\omega_{T}$. Thus, if $N_{j-1}(x) \notin \omega_{P^{\prime}}$ for some $P^{\prime} \in \mathbf{T}_{u}$ then the same condition will also be satisfied for all $P \in \mathbf{T}_{u}$ with $\omega_{P} \subset \omega_{P^{\prime}}$. We conclude that, for each $x$ and $j$, the bitiles that contribute to the sum are nested, their frequency intervals all contain some minimum frequency interval $\omega_{x, j}$ and are contained in some maximum frequency interval $\Omega_{x, j}$.

Now observe that the time intervals of these bitiles are also nested since they all contain $J$. Since the area of each bitile is fixed we conclude that for every $J \in \mathcal{J}$, $x \in J$ and $1 \leq j \leq K(x)$, there are some dyadic intervals $I_{x, j}^{\text {small }}$ and $I_{x, j}^{\text {large }}$ such that

$$
J \subset I_{x, j}^{\text {small }} \subsetneq I_{x, j}^{\text {large }}
$$

and

$$
\begin{aligned}
\left|\sum_{P \in \mathbf{T}_{u}, I_{P} \supsetneq J} \epsilon_{P} \epsilon_{P T}\left\langle f, w_{P_{d}}\right\rangle h_{I_{P}}(x) a_{j}(x) \mathbf{1}_{A(P, j)}(x)\right| \\
=\left|\sum_{P \in \mathbf{T}_{u}, I_{x, j}^{\text {small } \subsetneq I_{P} \subseteq I_{x, j}^{\text {large }}}} \epsilon_{P} \epsilon_{P T}\left\langle f, w_{P_{d}}\right\rangle h_{I_{P}}(x) a_{j}(x)\right| .
\end{aligned}
$$

In fact we will have that $I_{x, j}^{\text {small }}$ is the time interval corresponding to $\Omega_{x, j}$ and that $I_{x, j}^{\text {large }}$ is the time interval corresponding to $\omega_{x, j}$. From this it is also not hard to see that we also have the property $I_{x, j}^{\text {large }} \subsetneq I_{x, j+1}^{\text {small }}$ for each $j$. Using these 
observations and this notation we can now estimate

$$
\begin{aligned}
\left|\sum_{P \in \mathbf{T}_{u}, I_{P} \supsetneq J} \epsilon_{P} \epsilon_{P T}\left\langle f, w_{P_{d}}\right\rangle h_{I_{P}}(x) a_{j}(x) \mathbf{1}_{A(P, j)}(x)\right| \\
=\left|\sum_{P \in \mathbf{T}_{u}, I_{x, j}^{\text {small }} \subsetneq I_{P} \subseteq I_{x, j}^{\text {large }}} \epsilon_{P} \epsilon_{P T}\left\langle f, w_{P_{d}}\right\rangle h_{I_{P}}(x) a_{j}(x)\right| \\
\leq\left|a_{j}(x) w_{T_{u}}^{\infty}(x)\left(\mathbb{E}_{I_{x, j}^{\text {small }}}-\mathbb{E}_{I_{x, j}^{\text {large }}}\right)\left(\sum_{P \in \mathbf{T}_{u}} \epsilon_{P} \epsilon_{P T}\left\langle f, w_{P_{d}}\right\rangle h_{I_{P}}\right)(x)\right| \\
=\left|a_{j}(x)\left(\mathbb{E}_{I_{x, j}^{\text {small }}}-\mathbb{E}_{I_{x, j}^{\text {large }}}\right)\left(w_{T_{u}}^{\infty} \sum_{P \in \mathbf{T}_{u}} \epsilon_{P}\left\langle f, w_{P_{d}}\right\rangle w_{P_{d}}\right)(x)\right|,
\end{aligned}
$$

since $\left|w_{T_{u}}^{\infty}\right|=1$. The expectation operator $\mathbb{E}_{I}$ is defined in (2.1).

Remember that $\mathbb{E}_{\ell} f$ denotes the martingale of dyadic averages of $f$ with respect to dyadic intervals of length $2^{\ell}$. Summing over $j \in[1, K(x)]$ for which $N_{j}(x) \in \omega(J)$ and using Hölder's inequality we get

$$
\begin{aligned}
\left|F_{J, u}(x)\right| \leq & \mathbf{1}_{J}(x)|g(x)| \\
\times & \sum_{\substack{1 \leq j \leq K(x) \\
j: N_{j}(x) \in \omega(J)}}\left|a_{j}(x)\left(\mathbb{E}_{I_{x, j}^{\text {small }}}-\mathbb{E}_{I_{x, j}^{\text {large }}}\right)\left(w_{T_{u}}^{\infty} \sum_{P \in \mathbf{T}_{u}} \epsilon_{P}\left\langle f, w_{P_{d}}\right\rangle w_{P_{d}}\right)(x)\right| \\
\leq & \mathbf{1}_{J}(x)|g(x)|\left(\sum_{\substack{1 \leq j \leq K(x) \\
j: N_{j}(x) \in \omega(J)}}\left|a_{j}(x)\right|^{r^{\prime}}\right)^{1 / r^{\prime}} \\
& \times\left(\sum_{\substack{1 \leq j \leq K(x) \\
j: N_{j}(x) \in \omega(J)}}\left|\left(\mathbb{E}_{\ell_{j}}-\mathbb{E}_{\ell_{j}^{\prime}}\right)\left(w_{T_{u}}^{\infty} \tilde{f}\right)(x)\right|^{r}\right)^{1 / r},
\end{aligned}
$$

where $\ell_{1}, \ell_{2}, \ldots, \ell_{1}^{\prime}, \ell_{2}^{\prime}, \ldots$, are integers with $|J| \leq 2^{\ell_{1}}<2^{\ell_{1}{ }^{\prime}}<2^{\ell_{2}}<2^{\ell_{2}{ }^{\prime}}<\cdots<$ $2^{\ell_{j}}<2^{\ell_{j}^{\prime}}<\cdots$, and $\tilde{f}:=\sum_{P \in \mathbf{T}_{u}} \epsilon_{P}\left\langle f, w_{P_{d}}\right\rangle w_{P_{d}}$. Taking the supremum over all such choices of integers $\ell_{j}$ and $\ell_{j}^{\prime}$ for $1 \leq j \leq K$ and all positive integers $K$ and integrating over $J$ gives the estimate

$$
\int_{J}\left|F_{J, u}(x)\right|^{r^{\prime}} d x \leq \int_{J}|g(x)|^{r^{\prime}} \sum_{j: N_{j}(x) \in \omega(J)}\left|a_{j}(x)\right|^{r^{\prime}}\left\|\mathbb{E}_{k}\left(w_{T_{u}}^{\infty} \tilde{f}\right)(x)\right\|_{\mathcal{V}_{2^{k} \geq|J|}^{r^{\prime}}} d x
$$

Here we write

$$
\left\|b_{k}\right\| \mathcal{\nu}_{2^{k} \geq A}^{r}:=\sup _{K} \sup _{\substack{\ell_{0}<\ell_{1}<\cdots<\ell_{K} \\ 2^{\ell_{j}} \geq A, j=0, \ldots, K}}\left(\sum_{j=1}^{K}\left\|\beta_{\ell_{j+1}}-\beta_{\ell_{j}}\right\|^{r}\right)^{1 / r}
$$


The function $\left\|\mathbb{E}_{k} w_{T_{u}}^{\infty} \tilde{f}(\cdot)\right\|_{\mathcal{V}_{2^{k} \geq|J|}^{r}}$ is constant on $J$ thus

$$
\begin{aligned}
\left\|F_{J, u}\right\|_{L^{r^{\prime}\left(\mathbb{R}_{+}\right)}}^{r^{\prime}} \leq & \int_{J}\left\{|g(x)|^{r^{\prime}} \sum_{j: N_{j}(x) \in \omega(J)}\left|a_{j}(x)\right|^{r^{\prime}}\right. \\
& \left.\times\left(\frac{1}{|J|} \int_{J}\left\|\mathbb{E}_{k}\left(w_{T_{u}}^{\infty} \tilde{f}\right)(z)\right\|_{\mathcal{V}_{2^{k} \geq|J|}^{r}} d z\right)^{r^{\prime}}\right\} d x \\
\leq & |J| \operatorname{density}(\mathbf{T})^{r^{\prime}} \inf _{J}\left[M\left(\left\|\mathbb{E}_{k}\left(w_{T_{u}}^{\infty} \tilde{f}\right)(\cdot)\right\| \mathcal{V}^{r}\right)\right]^{r^{\prime}} \\
\leq & \operatorname{density}(\mathbf{T})^{r^{\prime}} \int_{J}\left[M\left(\left\|\mathbb{E}_{k}\left(w_{T_{u}}^{\infty} \tilde{f}\right)(\cdot)\right\| \mathcal{V}^{r}\right)(y)\right]^{r^{\prime}} d y
\end{aligned}
$$

where in the second inequality we also used Lemma 6.2, (iii). Here we remember that $M$ is the dyadic maximal operator defined in $\S 2$. Now we sum over $J \in \mathcal{J}$ and use the boundedness of $M$ on $L^{r^{\prime}}$ to get

$$
\begin{aligned}
\left(\sum_{J \in \mathcal{J}}\left\|F_{J, u}\right\|_{L^{r^{\prime}\left(\mathbb{R}_{+}\right)}}^{r^{\prime}}\right)^{1 / r^{\prime}} & \leq \operatorname{density}(\mathbf{T})\left(\int_{I_{T}}\left[M\left(\left\|\mathbb{E}_{k}\left(w_{T_{u}}^{\infty} \tilde{f}\right)(\cdot)\right\| \mathcal{V}^{r}(y)\right]^{r^{\prime}} d y\right)^{1 / r^{\prime}}\right. \\
& \lesssim \operatorname{density}(\mathbf{T})\left(\int\left[\left\|\mathbb{E}_{k}\left(w_{T_{u}}^{\infty} \tilde{f}\right)(y)\right\|_{\mathcal{V}^{r}}\right]^{r^{\prime}} d y\right)^{1 / r^{\prime}} \\
& \leq \operatorname{density}(\mathbf{T})\|\tilde{f}\|_{L^{r^{\prime}}\left(\mathbb{R}_{+}\right)},
\end{aligned}
$$

where in the last inequality we have used the vector-valued Lépingle inequality from Proposition 4.7. Observe that the use of Proposition 4.7 is allowed since $r^{\prime}<q^{\prime} \leq 2 \leq q<r$. Now Lemma 3.2 allows us to write

$$
\begin{aligned}
\|\tilde{f}\|_{L^{r^{\prime}\left(\mathbb{R}_{+}\right)}} & =\left\|\sum_{P \in \mathbf{T}_{u}} \epsilon_{P}\left\langle f, w_{P_{d}}\right\rangle w_{P_{d}}\right\|_{L^{r^{\prime}\left(\mathbb{R}_{+}\right)}}=\left\|\sum_{P \in \mathbf{T}_{u}} \epsilon_{P}\left\langle f w_{T_{u}}^{\infty}, h_{I_{P}}\right\rangle h_{I_{P}}\right\|_{L^{r^{\prime}}\left(\mathbb{R}_{+}\right)} . \\
(6.8) & \lesssim\left\|\sum_{P \in \mathbf{T}_{u}}\left\langle f w_{T_{u}}^{\infty}, h_{I_{P}}\right\rangle h_{I_{P}}\right\|_{L^{r^{\prime}}\left(\mathbb{R}_{+}\right)},
\end{aligned}
$$

the last inequality following by the UMD property of $X$. By another use of Lemma 3.2 and Hölder's inequality we have

$$
\begin{aligned}
\left\|\sum_{P \in \mathbf{T}_{u}}\left\langle f w_{T_{u}}^{\infty}, h_{I_{P}}\right\rangle h_{I_{P}}\right\|_{L^{r^{\prime}}\left(\mathbb{R}_{+}\right)} & =\left|I_{T}\right|^{1 / r^{\prime}}\left(\frac{1}{\left|I_{T}\right|} \int_{I_{T}}\left|\sum_{P \in \mathbf{T}_{u}}\left\langle f, w_{P_{d}}\right\rangle w_{P_{d}}(x)\right|^{r^{\prime}} d x\right)^{1 / r^{\prime}} \\
& \leq\left|I_{T}\right|^{1 / r^{\prime}}\left(\frac{1}{\left|I_{T}\right|} \int_{I_{T}}\left|\sum_{P \in \mathbf{T}_{u}}\left\langle f, w_{P_{d}}\right\rangle w_{P_{d}}(x)\right|^{q} d x\right)^{1 / q} \\
& \leq\left|I_{T}\right|^{1 / r^{\prime}} \operatorname{size}(\mathbf{T}),
\end{aligned}
$$

by the definition of size. Combining (6.7), (6.8), and (6.9), we get

$$
\left(\sum_{J \in \mathcal{J}}\left\|F_{J, u}\right\|_{L^{r^{\prime}}\left(\mathbb{R}_{+}\right)}^{r^{\prime}}\right)^{1 / r^{\prime}} \lesssim \operatorname{density}(\mathbf{T}) \operatorname{size}(\mathbf{T})\left|I_{T}\right|^{1 / r^{\prime}}
$$

which is the desired estimate. 


\section{The size and density lemmas}

Let $X$ be a UMD Banach space with tile type $q \geq 2$. In this section we recall the standard selection algorithms in terms of density and size. In terms of density we have:

Lemma 7.1 (Density lemma). Let $\mathbf{P}$ be a finite collection of bitiles and $\delta>0$. Define density with respect to some function $g: \mathbb{R}_{+} \rightarrow X^{*}$ with $|g| \leq \mathbf{1}_{E}$, where $E \subset \mathbb{R}_{+}$is a measurable set of finite measure, and a sequence $\left\{a_{j}(x)\right\}_{j}$ with $\sum_{j}\left|a_{j}(x)\right|^{r^{\prime}}=1$. For a given $\Delta>0$, there exists a decomposition

$$
\mathbf{P}=\mathbf{P}_{\text {sparse }} \bigcup \bigcup_{j} \mathbf{T}_{j}
$$

where each $\mathbf{T}_{j}$ is a tree,

$$
\operatorname{density}\left(\mathbf{P}_{\text {sparse }}\right) \leq \Delta
$$

and for all dyadic $J$ :

$$
\sum_{j: I_{T_{j}} \subset J}\left|I_{T_{j}}\right| \leq \Delta^{-r^{\prime}}|E \cap J|
$$

Remark 7.3. Estimate (7.2) encodes different types of information. Letting $J$ increase to $\mathbb{R}_{+}$, it shows that

$$
\left\|\sum_{j} \mathbf{1}_{I_{T_{j}}}\right\|_{1}=\sum_{j}\left|I_{T_{j}}\right| \leq \Delta^{-r^{\prime}}|E| .
$$

On the other hand, it also shows that

$$
\left\|\sum_{j} \mathbf{1}_{I_{T_{j}}}\right\|_{\mathrm{BMO}} \lesssim \sup _{J} \frac{1}{|J|} \sum_{j: I_{T_{j}} \subset J}\left|I_{T_{j}}\right| \leq \Delta^{-r^{\prime}} \sup _{J} \frac{|E \cap J|}{|J|}
$$

where the supremum is over all dyadic $J$ that contain at least one $I_{T_{j}}$.

Proof. We choose $\mathbf{P}_{\text {sparse }}$ to be as big as possible

$$
\mathbf{P}_{\text {sparse }}:=\left\{P \in \mathbf{P}: \sup _{P^{\prime} \geq P} \frac{1}{\left|I_{P^{\prime}}\right|} \int_{I_{P^{\prime}}}|g(x)|^{r^{\prime}} \sum_{j: N_{j}(x) \in \omega_{P^{\prime}}}\left|a_{j}(x)\right|^{r^{\prime}} d x \leq \Delta^{r^{\prime}}\right\} .
$$

For every $P \in \mathbf{P} \backslash \mathbf{P}_{\text {sparse }}$ there exists a bitile $P^{\prime}$ such that

$$
\left|I_{P^{\prime}}\right| \leq \Delta^{-r^{\prime}} \int_{I_{P^{\prime}}}|g(x)|^{r^{\prime}} \sum_{j: N_{j}(x) \in \omega_{P^{\prime}}}\left|a_{j}(x)\right|^{r^{\prime}} d x .
$$

Among the chosen bitiles $P^{\prime}$ we call $\left\{T_{k}\right\}_{k}$ the bitiles that are maximal with respect to the partial order ' $\leq$ '. Now set

$$
\mathbf{T}_{k}:=\left\{P \in \mathbf{P} \backslash \mathbf{P}_{\text {sparse }}: P \leq T_{k}\right\} .
$$


It is clear that $\mathbf{P} \backslash \mathbf{P}_{\text {sparse }}=\cup_{k} \mathbf{T}_{k}$. We have

$$
\begin{aligned}
\sum_{k: I_{T_{k}} \subset J}\left|I_{T_{k}}\right| & \leq \Delta^{-r^{\prime}} \sum_{k: I_{T_{k}} \subset J} \sum_{j} \int_{I_{T_{k}}}|g(x)|^{r^{\prime}}\left|a_{j}(x)\right|^{r^{\prime}} \mathbf{1}_{\left\{y: N_{j}(y) \in \omega_{T_{k}}\right\}}(x) d x \\
& \leq \Delta^{-r^{\prime}} \sum_{j} \sum_{k: I_{T_{k}} \subset J} \int_{I_{T_{k}} \cap E \cap\left\{y: N_{j}(y) \in \omega_{T_{k}}\right\}}\left|a_{j}(x)\right|^{r^{\prime}} d x .
\end{aligned}
$$

Now, for $j$ fixed, the sets $I_{T_{k}} \cap E \cap\left\{y: N_{j}(y) \in \omega_{T_{k}}\right\}, k \in \mathbb{Z}$, are all contained in $E \cap J$ and are pairwise disjoint for different $k$. Indeed if two of them intersected, say for $k_{1} \neq k_{2}$, then the corresponding bitiles $T_{k_{1}}$ and $T_{k_{2}}$ would also intersect, which contradicts their maximality. Summing first over $k$ and then over $j$ we get

$$
\sum_{k: I_{T_{k}} \subset J}\left|I_{T_{k}}\right| \leq \Delta^{-r^{\prime}} \sum_{j} \int_{E \cap J}\left|a_{j}(x)\right|^{r^{\prime}} d x \leq \Delta^{-r^{\prime}}|E \cap J|,
$$

since $\sum_{j}\left|a_{j}(x)\right|^{r^{\prime}}=1$.

For the selection by size we prove a version of the standard size selection algorithm.

Lemma 7.5 (Size lemma). Let $\mathbf{P}$ be a finite collection of bitiles and let $X$ a Banach space of tile type $q \geq 2$. Define size with respect to some function $f \in L^{q}\left(\mathbb{R}_{+} ; X\right)$. For a given $\varsigma>0$, there exists a disjoint decomposition

$$
\mathbf{P}=\mathbf{P}_{\text {small }} \bigcup \bigcup_{j} \mathbf{T}_{j}
$$

where each $\mathbf{T}_{j}$ is a tree,

$$
\operatorname{size}\left(\mathbf{P}_{\text {small }}\right) \leq \varsigma,
$$

and, for dyadic intervals $J$,

$$
\sum_{j: I_{T_{j}} \subset J}\left|I_{T_{j}}\right| \lesssim \varsigma^{-q}\left\|f \mathbf{1}_{J}\right\|_{L^{q}\left(\mathbb{R}_{+} ; X\right)}^{q} \leq \varsigma^{-q}|F \cap J| \quad \text { if }|f| \leq \mathbf{1}_{F} .
$$

Remark 7.7. As in Remark 7.3, estimate (7.6) implies

$$
\left\|\sum_{j} \mathbf{1}_{I_{T_{j}}}\right\|_{1} \lesssim \varsigma^{-q}|F|, \quad\left\|\sum_{j} \mathbf{1}_{I_{T_{j}}}\right\|_{\mathrm{BMO}} \lesssim \varsigma^{-q} \sup _{J} \frac{|F \cap J|}{|J|},
$$

where the supremum is over all dyadic $J$ that contain at least one $I_{T_{j}}$.

The selection algorithm is contained in Proposition 6.1 of [13], but we briefly outline it here for the reader's convenience.

Proof. For every tree $\mathbf{T}$ we set

$$
\Delta(\mathbf{T})^{q}:=\frac{1}{\left|I_{T}\right|} \int\left|\sum_{P \in \mathbf{T}_{u}}\left\langle f, w_{P_{d}}\right\rangle w_{P_{d}}(x)\right|^{q} d x,
$$


where $T$ is a top of $\mathbf{T}$. We iterate the following selection algorithm. Consider all maximal trees $\mathbf{T}$ inside $\mathbf{P}$ with $\Delta(\mathbf{T})>\varsigma$. Among them let $\mathbf{T}_{1}$ be one with top $T_{1}$ whose frequency interval $\omega_{T_{1}}$ has minimal center. Replace $\mathbf{P}$ by $\mathbf{P} \backslash \mathbf{T}_{1}$ and iterate. When no trees can be selected any longer the remaining collection, $\mathbf{P}_{\text {small }}$, by definition satisfies $\operatorname{size}\left(\mathbf{P}_{\text {small }}\right) \leq \varsigma$. Let $\left\{\mathbf{T}_{j}\right\}_{j}$ be the selected trees. The top time intervals $I_{T_{j}}$ of the selected trees can thus be estimated by

$$
\sum_{j: I_{T_{j}} \subset J}\left|I_{T_{j}}\right| \leq \frac{1}{\varsigma^{q}} \sum_{j: I_{T_{j}} \subset J}\left\|\sum_{P \in \mathbf{T}_{j, u}}\left\langle f, w_{P_{d}}\right\rangle w_{P_{d}}\right\|_{L^{q}\left(\mathbb{R}_{+} ; X\right)}^{q},
$$

and we can replace $f$ by $f \mathbf{1}_{J}$, since $w_{P_{d}}$ is supported on $I_{P} \subset I_{T_{j}} \subset J$. The collection $\mathcal{T}:=\left\{\mathbf{T}_{j, u}\right\}_{j}$ of the selected up-trees is a good collection by construction. Thus the tile type property of $X$ implies that the sum on the right-hand side of the previous estimate can be estimated by $\left\|f \mathbf{1}_{J}\right\|_{L^{q}\left(\mathbb{R}_{+} ; X\right)}^{q}$. We get

$$
\sum_{j: I_{T_{j}} \subset J}\left|I_{T_{j}}\right| \lesssim \varsigma^{-q}\left\|f \mathbf{1}_{J}\right\|_{L^{q}\left(\mathbb{R}_{+} ; X\right)}^{q}
$$

as desired.

Iterating the density and size lemmas we can write any finite collection as a union of trees.

Lemma 7.8. Let $\mathbf{P}$ be a finite collection of bitiles, and define size with respect to $f: \mathbb{R}_{+} \rightarrow X$ with $|f| \leq \mathbf{1}_{F}$ and density with respect to $g: \mathbb{R}_{+} \rightarrow X^{*}$ with $|g| \leq \mathbf{1}_{E}$. Then $\mathbf{P}$ admits a decomposition

$$
\mathbf{P}=\bigcup_{n \in \mathbb{Z}} \bigcup_{j} \mathbf{T}_{n, j} \cup \mathbf{P}_{\text {residual }}
$$

such that

$$
\operatorname{density}\left(\cup_{j} \mathbf{T}_{n, j}\right) \leq 2^{n / r^{\prime}}|E|^{1 / r^{\prime}}, \quad \operatorname{size}\left(\cup_{j} \mathbf{T}_{n, j}\right) \leq 2^{n / q}|F|^{1 / q}, \quad \sum_{j}\left|I_{\mathbf{T}_{n, j}}\right| \lesssim 2^{-n},
$$

and $\operatorname{density}\left(\mathbf{P}_{\text {residual }}\right)=\operatorname{size}\left(\mathbf{P}_{\text {residual }}\right)=0$.

The following bounds are available under additional assumptions:

(1) If $\inf _{x \in I_{P}} M\left(\mathbf{1}_{F}\right)(x) \leq|F| /|E| \leq 1$ for all $P \in \mathbf{P}$, then

$$
\left\|\sum_{j} \mathbf{1}_{I_{\mathbf{T}_{n, j}}}\right\|_{\mathrm{BMO}} \lesssim 2^{-n}|E|^{-1}
$$

(2) If $\inf _{x \in I_{P}} M\left(\mathbf{1}_{E}\right)(x) \leq|E| /|F| \leq 1$ for all $P \in \mathbf{P}$, then

$$
\left\|\sum_{j} \mathbf{1}_{I_{\mathbf{T}_{n, j}}}\right\|_{\mathrm{BMO}} \lesssim 2^{-n}|F|^{-1} .
$$


Proof. Since $\mathbf{P}$ is a finite collection of bitiles there exists some positive integer $n_{o}$ such that

$$
\operatorname{density}\left(\mathbf{P}^{\prime}\right) \leq 2^{n_{o} / r^{\prime}}|E|^{1 / r^{\prime}}, \quad \operatorname{size}\left(\mathbf{P}^{\prime}\right) \leq 2^{n_{o} / q}|F|^{1 / q}
$$

We apply Lemma 7.1 with $\Delta=2^{\left(n_{0}-1\right) / r^{\prime}}|E|^{1 / r^{\prime}}$ to write

$$
\mathbf{P}^{\prime}=\mathbf{P}_{1}^{\prime} \bigcup \bigcup_{j} \mathbf{T}_{j}
$$

with

$$
\operatorname{density}\left(\mathbf{P}_{1}^{\prime}\right) \leq 2^{\left(n_{o}-1\right) / r^{\prime}}|E|^{1 / r^{\prime}} \text { and } \sum_{j}\left|I_{T_{j}}\right| \leq\left(2^{\frac{n_{o}-1}{r^{\prime}}}|E|^{1 / r^{\prime}}\right)^{-r^{\prime}}|E|=2 \cdot 2^{-n_{o}} \text {. }
$$

We also have that

$$
\left\|\sum_{j} \mathbf{1}_{I_{T_{j}}}\right\|_{\mathrm{BMO}} \lesssim\left(2^{\frac{n_{o}-1}{r^{\prime}}}|E|^{1 / r^{\prime}}\right)^{-r^{\prime}} \sup _{J} \frac{|E \cap J|}{|J|} \lesssim 2^{-n_{o}}|E|^{-1} \sup _{J} \inf _{x \in J} M\left(\mathbf{1}_{E}\right)(x),
$$

where the supremum is over $J$ that contain at least one $I_{T_{j}}$, therefore at least one $I_{P}$ with $P \in \mathbf{P}$. The maximal term is always bounded by 1 , and under assumption (2) also by $|E| /|F|$.

We reduce the size of the collection in a similar fashion. We apply the size lemma with $\varsigma=2^{\left(n_{o}-1\right) / q}|F|^{1 / q}$ to $\mathbf{P}_{1}^{\prime}$ to write

$$
\mathbf{P}_{1}^{\prime}=\mathbf{P}_{1,1}^{\prime} \bigcup \bigcup_{j} \tilde{\mathbf{T}}_{j}
$$

with

$$
\operatorname{size}\left(\mathbf{P}_{1,1}^{\prime}\right) \leq 2^{\frac{n_{o}-1}{q}}|F|^{1 / q} \text { and } \sum_{j}\left|I_{\tilde{T}_{j}}\right| \lesssim\left(2^{\frac{n_{o}-1}{q}}|F|^{1 / q}\right)^{-q}\|f\|_{L^{q}\left(\mathbb{R}_{+} ; X\right)}^{q} \lesssim 2^{-n_{o}} .
$$

Under the additional assumptions, we also have that

$$
\left\|\sum_{j} \mathbf{1}_{I_{\tilde{T}_{j}}}\right\|_{\mathrm{BMO}} \lesssim\left(2^{\frac{n_{O}-1}{q}}|F|^{1 / q}\right)^{-q} \sup _{J} \frac{|F \cap J|}{|J|} \lesssim 2^{-n_{0}}|F|^{-1} \sup _{J} \inf _{x \in J} M\left(\mathbf{1}_{F}\right)(x),
$$

where the supremum is over all dyadic $J$ that contain at least one top $I_{\tilde{T}_{j}}$, hence at least one $I_{P}, P \in \mathbf{P}$. The maximal term is always bounded by 1 , and under the assumption (1) also by $\inf _{x \in J} M\left(\mathbf{1}_{F}\right)(x) \leq \inf _{x \in I_{P}} M\left(\mathbf{1}_{F}\right)(x) \lesssim|F| /|E|$.

Altogether, we find that $\left\{\mathbf{T}_{n_{o}, j}\right\}_{j}:=\left\{\mathbf{T}_{j}\right\}_{j} \cup\left\{\tilde{\mathbf{T}}_{j}\right\}_{j}$ satisfies

$$
\sum_{j}\left|I_{\mathbf{T}_{n_{o}, j}}\right| \lesssim 2^{-n_{o}}
$$

and

$$
\left\|\sum_{j} \mathbf{1}_{I_{\mathrm{T}_{n_{o}, j}}}\right\|_{\mathrm{BMO}} \lesssim \begin{cases}2^{-n_{0}}\left(|E|^{-1}+|F|^{-1} \cdot|F| /|E|\right) \lesssim 2^{-n_{o}}|E|^{-1}, & \text { under (1), } \\ 2^{-n_{0}}\left(|E|^{-1} \cdot|E| /|F|+|F|^{-1}\right) \lesssim 2^{-n_{o}}|F|^{-1}, & \text { under (2). }\end{cases}
$$


Since the density and size of any subcollection of $\mathbf{P}^{\prime}$ cannot increase, we also have

$$
\operatorname{density}\left(\cup_{j} \mathbf{T}_{n_{o}, j}\right) \leq 2^{n_{o} / r^{\prime}}|E|^{1 / r^{\prime}} \quad \text { and } \quad \operatorname{size}\left(\cup_{j} \mathbf{T}_{n_{o}, j}\right) \leq 2^{n_{o} / q}|F|^{1 / q} .
$$

We iterate this procedure until the residual collection has density and size equal to 0 .

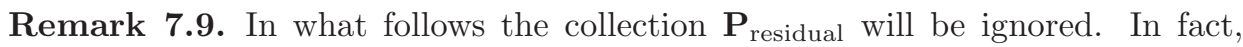
every $P \in \mathbf{P}_{\text {residual }}$ can be considered as a tree with a single bitile, and this tree will have both size and density equal to 0 . By the tree lemma these trivial trees do not contribute anything to the estimate for $C_{\mathbf{P}}$.

We record some additional size estimates that will allow us to obtain some initial control on the size of the collections we will consider.

Lemma 7.10. Let $\mathbf{P}$ be any collection of bitiles and define the density with respect to some function $g: \mathbb{R}_{+} \rightarrow X^{*}$ with $|g| \leq \mathbf{1}_{E}$ and the size with respect to some function $f: \mathbb{R}_{+} \rightarrow X$ with $|f| \leq \mathbf{1}_{F}$. We have that $\operatorname{density}(\mathbf{P}) \leq 1$ and $\operatorname{size}(\mathbf{P}) \lesssim 1$.

The following bounds are available under additional assumptions:

(1) If $\inf _{x \in I_{P}} M\left(\mathbf{1}_{F}\right)(x) \lesssim|F| /|E| \leq 1$ for all $P \in \mathbf{P}$, then

$$
\operatorname{size}(\mathbf{P}) \lesssim \frac{|F|}{|E|} .
$$

(2) If $\inf _{x \in I_{P}} M\left(\mathbf{1}_{E}\right)(x) \lesssim|E| /|F| \leq 1$ for all $P \in \mathbf{P}$, then

$$
\operatorname{density}(\mathbf{P}) \leq\left(\frac{|E|}{|F|}\right)^{1 / r^{\prime}} .
$$

Proof. The proof of the density estimate by 1 is completely trivial, while the proof of the size estimate by 1 is in Lemma 7.1 of [13], and relies on the UMD property of $X$.

Under the assumption (2), the density satisfies

$$
\begin{aligned}
\operatorname{density}(\mathbf{P}) & \leq \sup _{P \in \mathbf{P}} \sup _{P^{\prime} \geq P}\left(\frac{1}{\left|I_{P^{\prime}}\right|}\left|I_{P^{\prime}} \cap E\right|\right)^{1 / r^{\prime}} \\
& \leq \sup _{P \in \mathbf{P}} \inf _{x \in I_{P}} M\left(\mathbf{1}_{E}\right)(x)^{1 / r^{\prime}} \leq\left(\frac{|E|}{|F|}\right)^{1 / r^{\prime}} .
\end{aligned}
$$

Under the assumption (1), a standard argument using Lemmas 3.2 and 7.11 (below) gives the size bound asserted in this case; for the details of this argument see for example the proof of Lemma 7.3 in [13].

The following lemma, which we referred to above, is a BMO-type of estimate and is contained in Lemma 7.2 of [13].

Lemma 7.11. Let $\mathcal{J} \subseteq\left\{I \in \mathcal{D}: \inf _{y \in I} M f(x) \leq \lambda\right\}$ be a finite collection of dyadic intervals and let $K$ be a dyadic interval. Then, for all $1 \leq p<\infty$,

$$
\left\|\sum_{I \in \mathcal{J}, I \subseteq K}\left\langle f, h_{I}\right\rangle h_{I}\right\|_{L^{P}\left(\mathbb{R}_{+} ; X\right)} \leq \lambda|K|^{1 / p},
$$




\section{Proof of Proposition 5.6}

We need to prove that for every pair of measurable sets $E$ and $F$ with $|E|,|F|<+\infty$ there exists subsets $E^{\prime} \subset E$, and $F^{\prime} \subset F$ such that either $E^{\prime}=E$ or $F^{\prime}=F$, and

$$
\left|\left\langle C_{\mathbf{P}} f, g\right\rangle\right| \lesssim|F|^{1 / p}|E|^{1 / p^{\prime}}
$$

for $\max \left(q, p^{\prime}(q-1)\right)<r<\infty$ and for all $|f| \leq \mathbf{1}_{F^{\prime}}$ and $|g| \leq \mathbf{1}_{E^{\prime}}$. By dilation invariance we can assume that $1<|E| \leq 2$.

Case 1: $|\boldsymbol{F}| \leq$ 1. Let

$$
G:=\left\{M\left(\mathbf{1}_{F}\right)>4|F|\right\} .
$$

Then $|G| \leq 1 / 4$ by the weak $(1,1)$ bound on $M$ and thus $E^{\prime}:=E \backslash G$ can be taken as a major subset of $E$. We have

$$
\left\langle C_{\mathbf{P}} f, g\right\rangle=\sum_{P \in \mathbf{P}} \epsilon_{P}\left\langle f, w_{P_{d}}\right\rangle\left\langle w_{P_{d}} a_{P}, g\right\rangle=\sum_{P \in \mathbf{P}, I_{P} \nsubseteq G}+\sum_{P \in \mathbf{P}, I_{P} \subseteq G} .
$$

The second sum vanishes since each $w_{P_{d}}$ is supported on $I_{P} \subseteq G$ while $g$ is supported on $E^{\prime} \subseteq G^{c}$. Hence it suffices to consider any collection $\mathbf{P}^{\prime} \subseteq\{P \in \mathbf{P}$ : $\left.I_{P} \nsubseteq G\right\}$ and prove the corresponding bound for $C_{\mathbf{P}^{\prime}}$ in place of $C_{\mathbf{P}}$. Observe that for all $P \in \mathbf{P}^{\prime}$ we have that $I_{P} \nsubseteq G$ so that $\inf _{x \in I_{P}} M\left(\mathbf{1}_{F}\right)(x) \leq$ $\inf _{x \in I_{P} \backslash G} M\left(\mathbf{1}_{F}\right)(x) \leq 4|F|$ by the construction of $G$. Thus we are now in the situation that $\inf _{x \in I_{P}} M\left(\mathbf{1}_{F}\right)(x) \lesssim|F| \approx|F| /|E|$ for all $P \in \mathbf{P}^{\prime}$, for which several useful bounds were obtained in the previous section.

We now apply the decomposition given by Lemma 7.8 to the collection $\mathbf{P}^{\prime}$. Recalling the bounds density $\left(\mathbf{P}^{\prime}\right) \leq 1$ and $\operatorname{size}\left(\mathbf{P}^{\prime}\right) \lesssim|F|$ from Lemma 7.10, and the estimates of Lemma 7.8, we can write

$$
\operatorname{density}\left(\mathbf{T}_{n, j}\right) \lesssim \min \left(1,2^{n / r^{\prime}}\right), \operatorname{size}\left(\mathbf{T}_{n, j}\right) \leq \min \left(|F|, 2^{n / q}|F|^{1 / q}\right), \sum_{j}\left|I_{T_{n, j}}\right| \lesssim 2^{-n} .
$$

Applying Lemma 6.1 with $s=1$ we get

$$
\begin{aligned}
\left|\left\langle C_{\mathbf{P}} f, g\right\rangle\right| & =\left|\int \sum_{P \in \mathbf{P}} \epsilon_{P}\left\langle f, w_{P_{d}}\right\rangle w_{P_{d}}(x) a_{P}(x) g(x) d x\right| \\
& \leq \sum_{n \in \mathbb{Z}} \sum_{j} \int\left|\sum_{P \in \mathbf{T}_{n, j}} \epsilon_{P}\left\langle f, w_{P_{d}}\right\rangle w_{P_{d}}(x) a_{P}(x) g(x) d x\right| \\
& \lesssim \sum_{n \in \mathbb{Z}} \sum_{j}\left|I_{T_{n, j}}\right| \operatorname{size}\left(\mathbf{T}_{n, j}\right) \operatorname{density}\left(\mathbf{T}_{n, j}\right) \\
& \lesssim \sum_{n \in \mathbb{Z}} \min \left(|F|, 2^{n / q}|F|^{1 / q}\right) \min \left(1,2^{n / r^{\prime}}\right) \sum_{j}\left|I_{T_{n, j}}\right| \\
& \lesssim \sum_{n \in \mathbb{Z}} 2^{-n} \min \left(|F|, 2^{n / q}|F|^{1 / q}\right) \min \left(1,2^{n / r^{\prime}}\right) .
\end{aligned}
$$


We estimate the previous sum as follows:

$$
\begin{aligned}
& \left|\left\langle C_{\mathbf{P}} f, g\right\rangle\right| \lesssim|F|^{1 / q} \sum_{n: 2^{n} \leq|F|^{q / q^{\prime}}} 2^{n(1 / q-1 / r)}+|F| \sum_{n:|F|^{q / q^{\prime}<2^{n} \leq 1}} 2^{n\left(1 / r^{\prime}-1\right)}+|F| \sum_{n: 1<2^{n}} 2^{-n} \\
& \lesssim|F|^{1-(q-1) / r}+|F| \lesssim|F|^{1 / p} .
\end{aligned}
$$

In the last approximate inequality we used

$$
1-\frac{q-1}{r}=\frac{1}{p}+\frac{1}{p^{\prime}}-\frac{q-1}{r}>\frac{1}{p}
$$

and the hypothesis $|F| \leq 1$.

Case 2: $|\boldsymbol{F}| \geq 1$. Let $G:=\left\{M \mathbf{1}_{E}>8|F|^{-1}\right\}$, and set $F^{\prime}:=F \backslash G$. By the weak $(1,1)$ inequality for the dyadic maximal function $M$ we can conclude that $F^{\prime}$ is a major subset of $F$. Hence it suffices to consider any finite collection of bitiles $\mathbf{P}^{\prime} \subset\left\{P \in \mathbf{P}: I_{P} \not \subset G\right\}$ and prove the corresponding bound for $C_{\mathbf{P}^{\prime}}$. Thus the collection $\mathbf{P}^{\prime}$ has the property that $\inf _{x \in I_{P}} M\left(\mathbf{1}_{E}\right) \lesssim|E| /|F| \approx|F|^{-1}$ for all $P \in \mathbf{P}^{\prime}$, and several estimates from the previous section become available.

We again apply the decomposition given by Lemma 7.8 to the collection $\mathbf{P}^{\prime}$. Lemma 7.10 now provides the bounds $\operatorname{density}\left(\mathbf{P}^{\prime}\right) \lesssim|F|^{-1 / r^{\prime}}$ and $\operatorname{size}\left(\mathbf{P}^{\prime}\right) \lesssim 1$; combined with the estimates of Lemma 7.8, this leads to

$$
\operatorname{density}\left(\mathcal{T}_{n}\right) \lesssim \min \left(|F|^{-1 / r^{\prime}}, 2^{n / r^{\prime}}\right), \quad \operatorname{size}\left(\mathcal{T}_{n}\right) \leq \min \left(1,2^{n / q}\right), \quad \mathcal{T}_{n}:=\cup_{j} \mathbf{T}_{n, j}
$$

and

$$
\left\|\sum_{j} \mathbf{1}_{I_{\mathbf{T}_{n, j}, j}}\right\|_{1}=\sum_{j}\left|I_{\mathbf{T}_{n, j}}\right| \lesssim 2^{-n}, \quad\left\|\sum_{j} \mathbf{1}_{I_{\mathbf{T}_{n, j}, j}}\right\|_{\mathrm{BMO}} \lesssim 2^{-n}|F|^{-1} .
$$

Interpolation between the last two estimates gives the further bounds

$$
\left\|\sum_{j} \mathbf{1}_{I_{\mathbf{T}_{n, j}}}\right\|_{\tau} \lesssim 2^{-n}|F|^{-1 / \tau^{\prime}}, \quad \forall \tau \in[1, \infty) .
$$

For fixed $n$ we estimate

$$
\begin{aligned}
\left|\left\langle C_{\mathcal{T}_{n}} f, g,\right\rangle\right| & \leq \sum_{j} \int \mathbf{1}_{I_{T_{n}, j}}(x)\left|\sum_{P \in \mathbf{T}_{n, j}} \epsilon_{P}\left\langle f, w_{P_{d}}\right\rangle w_{P_{d}}(x) g(x) a_{P}(x)\right| d x \\
& \leq \int\left[\sum_{j} \mathbf{1}_{I_{T_{n, j}}}(x)\right]^{1 / r}\left[\sum_{j}\left|\sum_{P \in \mathbf{T}_{n, j}} \epsilon_{P}\left\langle f, w_{P_{d}}\right\rangle w_{P_{d}}(x) g[x) a_{P}(x)\right|^{r^{\prime}}\right]^{1 / r^{\prime}} d x \\
& \leq\left\|\left[\sum_{j} \mathbf{1}_{I_{T_{n, j}}}\right]^{1 / r}\right\|_{L^{\tau}} \cdot\left\|\left(\sum_{j}\left|\sum_{P \in \mathbf{T}_{n, j}} \epsilon_{P}\left\langle f, w_{P_{d}}\right\rangle w_{P_{d}} g a_{P}\right|^{r^{\prime}}\right)^{1 / r^{\prime}}\right\|_{L^{\tau^{\prime}}} \\
& =: A \cdot B,
\end{aligned}
$$


for $\tau \geq r$, which we will eventually choose 'large enough'. We note that the function inside the norm in $B$ is supported in $E$ and $\tau^{\prime}<r^{\prime}$. Thus, Hölder's inequality and Lemma 6.1 imply

$$
\begin{aligned}
B & \leq|E|^{1 / \tau^{\prime}-1 / r^{\prime}}\left(\sum_{j}\left\|\sum_{P \in \mathbf{T}_{n, j}} \epsilon_{P}\left\langle f, w_{P_{d}}\right\rangle w_{P_{d}} g a_{P}\right\|_{L^{r^{\prime}}}^{r^{\prime}}\right)^{1 / r^{\prime}} \\
& \lesssim \operatorname{size}\left(\mathcal{T}_{n}\right) \operatorname{density}\left(\mathcal{T}_{n}\right)\left(\sum_{j}\left|I_{T_{n, j}}\right|\right)^{1 / r^{\prime}} \\
& \lesssim \min \left(2^{n / q}|F|^{1 / q}, 1\right) \min \left(2^{n / r^{\prime}},|F|^{-1 / r^{\prime}}\right) 2^{-n / r^{\prime}} \\
& =\min \left(2^{n / q}|F|^{1 / q}, 2^{-n / r^{\prime}}|F|^{-1 / r^{\prime}}\right) .
\end{aligned}
$$

Using (8.2) with $\tau$ replaced by $\tau / r>1$ we estimate $A$ as follows:

$$
A=\left\|\left(\sum_{j} \mathbf{1}_{I_{T_{n, j}}}\right)^{1 / r}\right\|_{L^{\tau}}=\left\|\sum_{j} \mathbf{1}_{I_{T_{n, j}}}\right\|_{L^{\tau / r}}^{1 / r} \lesssim 2^{-n / r}|F|^{1 / \tau-1 / r} .
$$

By (8.3), (8.4), and (8.5), we get for all $\tau>r$ that

$$
\left|\left\langle C_{\mathcal{T}_{n}} f, g\right\rangle\right| \lesssim 2^{-n / r}|F|^{1 / \tau-1 / r} \min \left(2^{n / q}|F|^{1 / q}, 2^{-n / r^{\prime}}|F|^{-1 / r^{\prime}}\right) .
$$

Summing over $n \in \mathbb{Z}$ thus gives

$$
\begin{aligned}
\sum_{n \in \mathbb{Z}}\left|\left\langle C_{\mathcal{T}_{n}} f, g\right\rangle\right| & \lesssim \sum_{2^{n} \leq|F|^{-1}} 2^{n(1 / q-1 / r)}|F|^{1 / \tau-1 / r+1 / q}+\sum_{2^{n}>|F|^{-1}} 2^{-n}|F|^{1 / \tau-1} \\
& \lesssim|F|^{1 / \tau} \leq|F|^{1 / p}
\end{aligned}
$$

by taking $\tau \geq \max (p, r)$, and recalling that $|F| \geq 1$.

This concludes the proof of Proposition 5.6 and thereby of Theorems 5.2 and 1.1.

Remark 8.6. It is of some importance to note a subtle difference between the way we derive the estimates (8.1) in the present paper and the way such estimates have been derived in the scalar case in the literature. For example, in [20], [11], and [10], only the tops of trees produced by the size lemma are shown to satisfy the BMO estimate in (8.1). To deal with this problem the standard approach is to collect all the trees produced by the size and density lemmas and 'pass them through' the size lemma once more. This double application of the size lemma guarantees the BMO estimate. One however needs to argue that after the second application of the size lemma, the $L^{1}$-estimate persists. This is done by complementing the size lemma with a certain efficiency estimate, stating that if a collection is already a union of trees, $\mathbf{P}=\cup_{j} \mathbf{T}_{j}$, and the size lemma decomposes $\mathbf{P}$ into a union of some other trees $\left\{\tilde{\mathbf{T}}_{k}\right\}_{k}$, then $\sum_{k}\left|I_{\tilde{T}_{k}}\right| \lesssim \sum_{j}\left|I_{T_{j}}\right|$; that is, the size lemma is shown to be the most efficient algorithm in selecting trees and their tops when decomposing $\mathbf{P}$. In the vector-valued case we have not managed to produce such an efficiency estimate for the size lemma, which is strongly dependent on the tile type of the Banach space $X$. Instead, we directly produce BMO estimates, both for trees selected by size, as well as for the ones selected by density. 


\section{Interpolation}

In this section we discuss the proof of Theorem 1.4. This is a simple interpolation argument between the Hilbert space-valued case for the $r$-variation and the UMD valued case for the $\infty$-variation, that is the main result from [13].

Proof of Theorem 1.4. Let $X$ be a UMD Banach space of the form $X=[Y, H]_{\theta}$ for some $0<\theta<1$ and set $q=: 2 / \theta$. From [13] we have that the Carleson operator maps $L^{p}([0,1) ; X)$ into itself for all $1<p<\infty$. This can be rewritten in the form

$$
\left\|S_{N} f\right\|_{L^{p}\left([0,1) ; \ell^{\infty}(X)\right)} \lesssim\|f\|_{L^{p}([0,1) ; X)}, \quad 1<p<\infty .
$$

On the other hand we have for any Hilbert space $H$ the following variation norm Carleson theorem:

$$
\left\|S_{N} f\right\|_{L^{p}\left([0,1) ; \mathcal{V}^{s}(H)\right)} \lesssim\|f\|_{L^{p}([0,1) ; H)}, \quad s>2, \quad s^{\prime}<p<\infty .
$$

This follows, for example, from Theorem 1.3, although in the special case of a Hilbert space one could just repeat the scalar proof. We first use the reiteration theorem, as in Theorem 3.5.3 of [1], to write

$$
X=[Y, H]_{\theta}=\left[[Y, H]_{\delta}, H\right]_{\omega},
$$

with $\theta=(1-\omega) \delta+\omega$. In our considerations one should think of $\delta \rightarrow 0$. Now fix $1<p<\infty$ and interpolate between $L^{p}\left([0,1) ; \ell^{\infty}\left([Y, H]_{\delta}\right)\right)$ and $L^{p}\left([0,1) ; \mathcal{V}^{s}(X)\right)$. As in p. 501 of [23], we have

$$
\left[\ell^{\infty}\left([Y, H]_{\delta}\right), \mathcal{V}^{s}(H)\right]_{\omega} \subset \mathcal{V}^{s_{\omega}}\left(\left[[Y, H]_{\delta}, H\right]_{\omega}\right)=\mathcal{V}^{s_{\omega}}(X),
$$

where

$$
\frac{1}{s_{\omega}}=\frac{1-\omega}{\infty}+\frac{\omega}{s} \Longleftrightarrow s_{\omega}=\frac{s}{\omega},
$$

and $\theta=(1-\omega) \delta+\omega$. From this we get that

$$
\left\|S_{N} f\right\|_{L^{p}\left([0,1) ; \mathcal{V}^{r}(X)\right)} \lesssim\|f\|_{L^{p}([0,1) ; X)}
$$

whenever $r>2 / \theta=q$ and $r>p^{\prime} q / 2$.

Acknowledgment. We would like to thank the anonymous referee for an expert reading that resulted in several improvements throughout the paper.

\section{References}

[1] Bergh, J. And Löfström, J.: Interpolation spaces. An introduction. Grundlehren der Mathematischen Wissenschaften 223, Springer-Verlag, Berlin, 1976.

[2] Billard, P.: Sur la convergence presque partout des séries de Fourier-Walsh des fonctions de l'espace $L^{2}(0,1)$. Studia Math. 28 (1966/1967), 363-388. 
[3] Campbell, J. T., Jones, R. L., Reinhold, K. and Wierdl, M.: Oscillation and variation for the Hilbert transform. Duke Math. J. 105 (2000), no. 1, 59-83.

[4] Campbell, J. T., Jones, R. L., Reinhold, K. and Wierdl, M.: Oscillation and variation for singular integrals in higher dimensions. Trans. Amer. Math. Soc. 355 (2003), no. 5, 2115-2137 (electronic).

[5] Carleson, L.: On convergence and growth of partial sums of Fourier series. Acta Math. 116 (1966), 135-157.

[6] Christ, M. And Kiselev, A.: Scattering and wave operators for one-dimensional Schrödinger operators with slowly decaying nonsmooth potentials. Geom. Funct. Anal. 12 (2002), no. 6, 1174-1234.

[7] Demeter, C.: Pointwise convergence of the ergodic bilinear Hilbert transform. Illinois J. Math. 51 (2007), no. 4, 1123-1158.

[8] Demeter, C., Lacey, M. T., Tao, T. and Thiele, C.: Breaking the duality in the return times theorem. Duke Math. J. 143 (2008), no. 2, 281-355.

[9] Do, Y., Oberlin, R. And Palsson, E. A.: Variational bounds for a dyadic model of the bilinear Hilbert transform. Illinois J. Math. 57 (2013), no .1, 105-119.

[10] Do, Y. And LACEY, M.: Weighted bounds for variational Fourier series. Studia Math. 211 (2012), no. 2, 153-190.

[11] Do, Y., Oberlin, R. And Palsson, E. A.: Weighted bounds for variational WalshFourier series. J. Fourier Anal. Appl. 18 (2012), no. 6, 1318-1339.

[12] Hytönen, T. P. And Lacey, M. T.: Pointwise convergence of vector-valued Fourier series. Math. Ann. 357 (2013), no. 4, 1329-1361.

[13] Hytönen, T. P. And Lacey, M. T.: Pointwise convergence of Walsh-Fourier series of vector-valued functions. Preprint, arXiv: 1202.0209, 2012.

[14] Hytönen, T. P., Lacey, M. T. And Parissis, I.: The vector valued quartile operator. Collect. Math. 64 (2013), no. 3, 427-454.

[15] Jones, R. L., Seeger, A. And Wright, J.: Strong variational and jump inequalities in harmonic analysis. Trans. Amer. Math. Soc. 360 (2008), no. 12, 6711-6742.

[16] Lacey, M. AND Thiele, C.: A proof of boundedness of the Carleson operator. Math. Res. Lett. 7 (2000), no. 4, 361-370.

[17] LÉPingle, D.: La variation d'ordre $p$ des semi-martingales. Z. Wahrscheinlichkeitstheorie und Verw. Gebiete 36 (1976), no. 4, 295-316.

[18] Oberlin, R.: Bounds on the Walsh model for $M^{q, *}$ Carleson and related operators. Rev. Mat. Iberoam. 29 (2013), no. 3, 829-857.

[19] Oberlin, R.: Estimates for compositions of maximal operators with singular integrals. Canad. Math. Bull. 56 (2013), no. 4, 801-813.

[20] Oberlin, R., Seeger, A., Tao, T., Thiele, C. and Wright, J.: A variation norm Carleson theorem. J. Eur. Math. Soc. (JEMS) 14 (2012), no. 2, 421-464.

[21] Parcet, J., Soria, F. And Xu, Q.: On the growth of vector-valued Fourier series. Indiana Univ. Math. J. 62 (2013), no. 6, 1765-1784.

[22] Pisier, G.: Martingales with values in uniformly convex spaces. Israel J. Math. 20 (1975), no. 3-4, 326-350. 
[23] Pisier, G. And XU, Q.: The strong $p$-variation of martingales and orthogonal series. Probab. Theory Related Fields 77 (1988), no. 4, 497-514.

[24] Rubio de Francia, J. L.: Fourier series and Hilbert transforms with values in UMD Banach spaces. Studia Math. 81 (1985), no. 1, 95-105.

[25] Rubio de Francia, J. L.: Martingale and integral transforms of Banach space valued functions. In Probability and Banach spaces (Zaragoza, 1985), 195-222. Lecture Notes in Math. 1221, Springer, Berlin, 1986.

[26] Silva, P.: Vector valued inequalities for families of bilinear Hilbert transform and applications to bi-parameter problems. Preprint, arXiv: 1203.3251v1, 2012.

[27] Ma, T., Torrea, J. L. And Xu, Q.: Weighted variation inequalities for differential operators and singular integrals. Preprint, arXiv: 1301.6859, 2013.

[28] Thiele, C.: Wave packet analysis. CBMS Regional Conference Series in Mathematics 105, American Mathematical Society, Providence, RI, 2006.

[29] Weisz, F.: Almost everywhere convergence of Banach space-valued Vilenkin-Fourier series. Acta Math. Hungar. 116 (2007), no. 1-2, 47-59.

Received September 20, 2012; revised October 9, 2013.

Tuomas P. Hytönen: Department of Mathematics and Statistics, P. O. Box 68 (Gustaf Hällströmin katu 2b), FI-00014 University of Helsinki, Finland.

E-mail: tuomas.hytonen@helsinki.fi

Michael T. Lacey: School of Mathematics, Georgia Institute of Technology, Atlanta GA 30332, USA.

E-mail: lacey@math.gatech.edu

IoAnnis Parissis: Department of Mathematics, P. O. Box 11100 (Otakaari 1 M), FI-00076 Aalto University, Finland.

E-mail: ioannis.parissis@gmail.com

T.H. and I.P. are supported by the European Union through the ERC Starting Grant "Analytic-probabilistic methods for borderline singular integrals". T. H. is also supported by the Academy of Finland, grants 130166 and 133264 and I. P. also supported by the Academy of Finland, grant 138738. M. L. supported in part by the NSF grant 0968499, and a grant from the Simons Foundation (\#229596 to Michael Lacey). 